\title{
Nuclear prolate-shape dominance with the Woods-Saxon potential
}

\author{
Satoshi Takahara \\ Kyorin University, School of Medicine, Mitaka, Tokyo 181-8611, Japan \\ Naoki Tajima \\ Department of Applied Physics, University of Fukui, 3-9-1 Bunkyo, Fukui 910-8507, Japan \\ Yoshifumi R. Shimizu \\ Department of Physics, Graduate School of Science, Kyushu University, Fukuoka 812-8581, Japan
}

(Dated: July 6, 2018)

\begin{abstract}
We study the prolate-shape predominance of the nuclear ground-state deformation by calculating the masses of more than two thousand even-even nuclei using the Strutinsky method, modified by Kruppa, and improved by us. The influences of the surface thickness of the single-particle potentials, the strength of the spin-orbit potential, and the pairing correlations are investigated by varying the parameters of the Woods-Saxon potential and the pairing interaction. The strong interference between the effects of the surface thickness and the spin-orbit potential is confirmed to persist for six sets of the Woods-Saxon potential parameters. The observed behavior of the ratios of prolate, oblate, and spherical nuclei versus potential parameters are rather different in different mass regions. It is also found that the ratio of spherical nuclei increases for weakly bound unstable nuclei. Differences of the results from the calculations with the Nilsson potential are described in detail.

PACS numbers: 21.10.Ft, 21.10.Gv, 21.10.Ky, 21.10.Pc
\end{abstract}

\section{INTRODUCTION}

It has long been known that most of the deformed nuclei have prolate rather than oblate shapes [1]. This prolate-shape dominance is the subject of this paper, in which we examine the effects of several important factors to increase or decrease such preference for prolate shapes.

The properties of a nucleus are completely determined by the Hamiltonian for quantum mechanical many-body systems consisting of nucleons. From this point of view, a possible direction of research for the origin of the prolateshape dominance is to change artificially each term of the Hamiltonian and examine its effect on the prolateshape dominance. The most successful outcome of such a study would be a discovery of a direct correspondence between the prolate-shape dominance and a specific term of the elementary nucleon-nucleon interaction, in an analogous fashion as the tensor force causes a mixture of the $d$ wave in the wavefunction of a deuteron. However, nobody knows whether there exists such a simple relation.

In mean-field approximations all the influences of the many-body Hamiltonian on the nuclear shape are conveyed through the mean-field (one-body) Hamiltonian. Hence, one can seek the origin of the prolate-shape dominance in the properties of the mean field, instead. If one also adopts the macroscopic-microscopic method [2], which uses only the single-particle level density, one can determine the nuclear shape starting from the mean-field Hamiltonian without referring to the underlying manybody Hamiltonian. This is the choice of this paper. One can reasonably expect that the linkage from the meanfield Hamiltonian to the nuclear shape may be simpler than those from the many-body Hamiltonian. Moreover, the former linkage has an established route to understand in terms of classical periodic orbits [3, 4], while the latter has none presently.

We have been seeking the origin of the prolate-shape dominance in the one-body potentials in the macroscopicmicroscopic framework. In Ref. [5], we employed the Nilsson potential [6] and calculated the ratio of prolate-shape nuclei among $\sim 1800$ even-even nuclei as a function of the strengths of the $l^{2}$ term and the spin-orbit term. We found that the ratio of prolate nuclei oscillates versus the strength of the spin-orbit term when the $l^{2}$ term has the standard strength. This oscillation disappears when the $l^{2}$ potential is weaker. The amplitude of the oscillation is quite large. The ratio of prolate-shape nuclei oscillates between $\sim 40 \%$ (at $\pm 40 \%$ strengths of the standard) and $\geq 80 \%$ (at $\pm 100 \%$ and $0 \%$ strengths). This result indicates a strong interference between the effects of the two terms of the potential.

However, this interference might be exaggerated because of the affinity of the two terms in the sense that both of them include the orbital angular momentum operator $l$. The $l^{2}$ term is an approximation to the radial profile of the central potential like a square well. In a letter 7], we replaced the Nilsson potential with the WoodsSaxon (WS) potential to eliminate the $l^{2}$ potential. As a result, we still find the oscillation, although its amplitude was decreased by a factor $\sim \frac{1}{3}$.

Employment of the WS potential required various modifications of the method [8]. First, the WS potential has a continuum spectrum, which cannot be treated by the standard Strutinsky method. This problem was solved by the Kruppa's prescription [9] to subtract the level density of the free-particle spectrum. Concerning 
the remaining problem of the plateau condition, we invented the reference density method [8] to make the shell energy insensitive to the smearing width. Second, the BCS equation with a constant pair-scattering matrix element $G$ also suffers from the continuum spectrum. We extended the Kruppa's prescription to the paring to subtract the contributions from free-particle spectrum in the BCS equation. This treatment also improves the calculation of the nuclear radius [10]. Third, available parameter sets of the WS potentials are not consistent with the macroscopic part especially for nuclei far from the $\beta$-stability line. We presented a method based on the Thomas-Fermi approximation to adjust the depth of the central potential to reproduce the drip lines predicted by the macroscopic energy.

In this paper, we show and discuss the results of the calculations with the WS potential in much more detail than in the letter [7]. We try not a single but six parameter sets of the WS potential. We confirm the convergence of the results versus the size of the truncated oscillator basis for the diagonalization of the single-particle Hamiltonian. We show that different definitions of the ratio of prolate-shape nuclei do not change the conclusions. We also consider the effects of changing the strength of the pairing interaction, which we studied so far only for the Nilsson potential in Ref. [11]. We show the distribution of prolate and oblate nuclei in the nuclear chart for various modified potentials and confirm the independence of the main conclusions from the choice of potentials.

In the rest of the introduction, we survey the earlier works.

For the anisotropic harmonic oscillator potential, which is the most basic approximation to the one-nucleon potential, prolate (oblate) shapes tend to have lower energies than oblate (prolate) ones when the major shell is less (more) than half filled because the particles (holes) in the low $\Omega$ orbitals drives strongly the nuclear shape toward prolate (oblate) direction. Here, $\Omega$ is the projection of the single-particle angular momentum onto the symmetry axis of the mean-field potential. This suggests an approximately equal number of prolate and oblate nuclei [1]. Indeed, our quantitative estimation [5] showed that the ratio of prolate nuclei among deformed nuclei is $55 \%$ for a harmonic oscillator potential, while the experimental ratio of prolate nuclei seems much larger.

The earliest attempt to explain the prolate-shape preference is a work by Lemmer and Weisskopf 12]. A term $\propto r^{4}$ was added to the harmonic oscillator potential to steepen the wall. Then it overrode the normal tendency of the oscillator potential to deform into an oblate shape for a more-than-half-filled major shell. It suggests that the origin of the prolate-shape dominance is the radial profile of the potential.

However, there was also an opinion that the origin is the spin-orbit potential. For example, Ref. [1] states that it is the spin-orbit potential which breaks the even situation by weakening the oblate-shape shell effect in $s d$ shell nuclei.
The truth has turned out [5] that both of the two salient features of the nuclear single-particle potential, the square-well like radial profile and the spin-orbit potential, play essential roles to give rise to the prolateshape dominance. The dominance can be equally reproduced with and without the spin-orbit potential but it does not deny the participation of the spin-orbit potential, because half-strength spin-orbit potential destroys the dominance.

The relation between potential and spectrum can be understood in terms of classical periodic orbits. Putting aside the spin-orbit potential, Frisk analyzed classical periodic orbits in an ellipsoidal cavity and found that the radial dependence is an origin of the prolate-shape dominance [3]. The strength of the shell effect at the Fermi surface changes strongly in the prolate side while it stays almost constant in the oblate side as a function of the magnitude of deformation. As the classical periodic orbit responsible for this asymmetry between prolate and oblate shapes, Frisk payed attention to a triangular orbit in the meridian plane whose period changes little in the oblate side owing to the volume conservation condition.

Arita used a power-law potential $\propto r^{\alpha}[4]$, which can interpolate continuously between the square-well potential and the harmonic oscillator potentials, for the same kind of analysis and obtained essentially the same conclusion as Frisk [4]. Concerning the classical periodic orbits responsible for the prolate-shape dominance, Arita insisted the importance of bridge-orbit bifurcations. He is also planning to treat the spin-orbit potential in the periodic orbit theory [13].

Hamamoto and Mottelson presented a plain explanation to the fact that the level density in the Fermi level changes little in the oblate side [14]. In the Nilsson diagram of single-particle spectrum, the degenerated levels belonging to a subshell at spherical shape fans out (spreads) versus increasing magnitude of deformation in both prolate and oblate sides. However, this fanning out is small for levels with low $\Lambda$ (the projection of orbital angular momentum onto the symmetry axis) in the oblate side because they are pushed down by many orbitals with the same quantum number belonging to upper major shells. Since the same spectrum leads to the same value of the shell effect, no energy can be gained by deforming into oblate shapes.

However, Arita [4] found a counterexample to this explanation. For a low-power potential $\propto r^{1.1}$, the fanning is suppressed for low- $\Lambda$ orbitals in prolate shapes, while he found no oblate-shape dominance. Therefore, the explanation in terms of fanning applies indeed to nuclear-like potentials but probably not to different types of potentials. We think that the reason for this limitation is that the explanation in terms of the fanning mechanism does not explicitly consider the volume conservation condition which played an essential role in Frisk's argument [3].

Finally, we mention two other factors which have minor effects to prefer prolate shapes.

One is the Coulomb interaction, which tends to elon- 
gate the nuclear shape rather than flatten it in order to diminish positive electrostatic potential energy between protons. However, this effect does not seem to play a decisive role to give rise to the prolate-shape dominance because it increases as $Z^{2}$ and is strongest in heavy nuclei while the actual dominance is already very clear in middle-weight nuclei. This effect will be revisited in our future paper for more detailed studies.

The other is the angular-momentum projection of mean-field solutions into zero or low-spin spin states, which has a tendency to lower the energy of prolate states relative to that of oblate ones, because the former have generally larger moments of inertia than the latter. This effect seems to have already been argued by Zickendraht [15] as the difference of the volume element for the collective coordinates between prolate and oblate shapes, which is originated in the difference of the available configuration space in the spherical shell model. However, mean-field models and their phenomenological interactions are determined to reproduce the experiments without angular momentum projection and thus it is widely believed that they includes the effects of the projection. A quantitative examination of this belief will be a subject of our future study.

In Sec. II we explain about the potentials, our theoretical framework, some practical information of our calculations, and definitions of the ratio of prolate nuclei. In Sec. III] we give the results and discussions on them. In Sec. IV] we summarize the conclusions of this paper.

\section{METHODS}

We use the Woods-Saxon-Strutinsky 16 and the Nilsson-Strutinsky methods [6] to calculate the nuclear energy surface in the plane spanned by the axially symmetric quadrupole and hexadecapole deformation parameters and to determine the shape of the ground state of even-even nuclei from Oxygen to superheavy elements between drip lines. For both methods, the single-particle Hamiltonian is a sum of a free kinetic energy and a potential energy,

$$
H=\frac{p^{2}}{2 m}+V
$$

where $m$ is the bare nucleon mass (the average of a proton and a neutron masses). The Nilsson and the WS potentials used for $V$ are described in Secs. IA and ПB, respectively. The latter potential has a continuum part in the spectrum, which causes difficulties in the standard version of the Strutinsky method. Remedies we have chosen [8] are summarized shortly in Sec. [IC] Precision and other practical aspects of numerical computations are described in Secs. IID and IIE. The quantity which we study intensively in this paper is introduced in Sec. IIF.

\section{A. The Nilsson potential}

The potential of the Nilsson model [30] is expressed as

$$
\begin{gathered}
V(\boldsymbol{r})=\frac{m}{2}\left(\omega_{\perp}^{2} x^{2}+\omega_{\perp}^{2} y^{2}+\omega_{z}^{2} z^{2}\right)+\hbar \stackrel{\circ}{\omega}_{0} r_{t}^{2} \sqrt{\frac{4 \pi}{9}} \epsilon_{4} Y_{40}\left(\hat{\boldsymbol{r}}_{t}\right) \\
-2 f_{l s} \kappa_{N} \hbar \stackrel{\circ}{\omega}_{0} \boldsymbol{l}_{t} \cdot \boldsymbol{s}-f_{l l} \kappa_{N} \mu_{N} \hbar \stackrel{\circ}{\omega}_{0}\left(\boldsymbol{l}_{t}^{2}-\left\langle\boldsymbol{l}_{t}^{2}\right\rangle_{N}\right),
\end{gathered}
$$

where $\omega_{\perp}$ and $\omega_{z}$ are related to a quadrupole deformation parameter $\epsilon_{2}$ through $\omega_{\perp}=\omega_{0}^{\circ}\left(1+\frac{1}{3} \epsilon_{2}\right)$ and $\omega_{z}=\omega_{0}^{\circ}$ $\left(1-\frac{2}{3} \epsilon_{2}\right)$ with $\stackrel{\circ}{\omega}_{0}$ determined by the condition of a volume conservation $\omega_{\perp}^{2} \omega_{z}=\stackrel{\circ}{\omega}_{0}^{3}$. Denoted by $\epsilon_{4}$ is a hexadecapole deformation parameter. Orbital and spin angular momenta are denoted as $\boldsymbol{l}$ and $\boldsymbol{s}$, respectively and the subscript $t$ means the usage of the stretched coordinates. The third term is a spin-orbit potential, while the fourth term is called the $l^{2}$ potential. The latter term proportional to the square of the orbital angular momentum is used to simulate roughly the change of the radial profile of the central potential from $\propto r^{2}$ of the harmonic oscillator. With a standard negative strength of the fourth term $\left(-f_{l l} \kappa_{N} \mu_{N}\right)$ and without the spin-orbit potential, the spectrum is modified from that of the harmonic oscillator toward that of the square-well potential.

We adopt the standard values given in Table 1 of Ref. 17] for the parameters $\kappa_{N}$ and $\mu_{N}$ which are dependent on the harmonic oscillator shell $N_{\text {osc }}$. By changing the set of values of the multipliers $\left(f_{l s}, f_{l l}\right)$ from the standard values $(1,1)$, one can examine how the combination of these two potentials affects the prolate-shape dominance. For example, $f_{l s}=0$ (1) means no (standard) spin-orbit potential, while $f_{l l}=0$ (1) corresponds to harmonic-oscillator (standard) radial profile of the central potential.

In this paper, we calculate the ratio of prolate nuclei for $31 \times 16=496$ kinds of artificially modified Nilsson potentials defined by the combination of values of the multipliers $\left(f_{l s}, f_{l l}\right)$ in ranges $-1.5 \leq f_{l s} \leq 1.5$ and $0 \leq$ $f_{l l} \leq 1.5$ with $\Delta f_{l s}=\Delta f_{l l}=0.1$.

As a footnote, the $\epsilon_{\lambda}$-parametrization of nuclear deformation in the Nilsson potential is different from the conventional $\beta_{\lambda}$-parametrization based on the expansion of nuclear radius in terms of the spherical harmonics [18]. Including only the $\lambda=2$ deformation and requiring the ratio of axes is the same between the two kinds of parametrizations, as an example, one obtains $\epsilon_{2} \cong 0.95 \beta_{2}$ for small deformations while $\epsilon_{2} \cong 1.14 \beta_{2}$ for the ratio $2: 1$ of the ideal superdeformation.

In this paper, we describe quantities related to shapes in term of $\beta_{2}$. For example, we give the thresholds to distinguish prolate or oblate shapes from the spherical shape in terms of the values of $\beta_{2}$. For the solutions of the Nilsson-Strutinsky method, we apply these thresholds directory to $\epsilon_{2}$, not calculating the value of $\beta_{2}$ corresponding to the value of $\epsilon_{2}$, for the sake of simplicity. 


\section{B. The Woods-Saxon potential}

The Woods-Saxon (WS) potential is a finite-depth potential having a flat central and a steep surface parts. It is no doubt a better approximation to the central part of the nuclear potential $V_{\mathrm{CE}}$ than the Nilsson potential. The spin-orbit potential $V_{\mathrm{SO}}$ is also reasonably expressed using the gradient of this form of a potential. The Coulomb potential $V_{\mathrm{CO}}$ should be added for protons. Then, the sum of these three potentials, which we also call the WS potential, is expressed as

$$
V=V_{\mathrm{CE}}+V_{\mathrm{SO}}+\frac{1}{2}\left(1-\tau_{3}\right) V_{\mathrm{CO}},
$$

where $\tau_{3}$ is the third component of the nucleon's isospin multiplied by 2 ( 1 for neutrons and -1 for protons),

$$
\begin{aligned}
V_{\mathrm{CE}} & =V_{\mathrm{WS}}\left(\boldsymbol{r} ; V_{\mathrm{OCE}}, \kappa_{\mathrm{CE}}, R_{0 \mathrm{CE}}, f_{a} a_{\mathrm{CE}}, \boldsymbol{\beta}\right), \\
V_{\mathrm{SO}} & =f_{l s} \lambda_{\mathrm{SO}}\left(\frac{\hbar}{2 m_{\mathrm{red}} c}\right)^{2} \times \\
& {\left[\nabla V_{\mathrm{WS}}\left(\boldsymbol{r} ; V_{\mathrm{OCE}}, \kappa_{\mathrm{SO}}, R_{0 \mathrm{SO}}, f_{a} a_{\mathrm{SO}}, \boldsymbol{\beta}\right)\right] . } \\
& \left(\boldsymbol{\sigma} \times \frac{1}{i} \nabla\right) .
\end{aligned}
$$

In Eq. (5), $m_{\text {red }}=\frac{A-1}{A} m$ with $m$ being the bare nucleon mass is the reduced mass, and $\boldsymbol{\sigma}$ is the Pauli matrix for the nucleon's spin. The function $V_{\mathrm{ws}}$ is defined by

$$
\begin{aligned}
& V_{\mathrm{WS}}\left(\boldsymbol{r} ; V_{0}, \kappa, R_{0}, a, \boldsymbol{\beta}\right) \\
& =-V_{0}\left[1 \pm \kappa \frac{N-Z}{A}\right] \frac{1}{1+\exp \left[\operatorname{dist}_{\Sigma}(\boldsymbol{r}, \boldsymbol{\beta}) / a\right]} .
\end{aligned}
$$

where $\operatorname{dist}_{\Sigma}(\boldsymbol{r}, \boldsymbol{\beta})$ is the perpendicular distance between the point $\boldsymbol{r}$ and the surface $\Sigma$ (taken with the minus sign inside the nucleus).

For axially symmetric nuclear shapes, the surface $\Sigma$ is defined by

$$
R\left(\theta ; R_{0}, \boldsymbol{\beta}\right)=R_{0} c_{v}(\boldsymbol{\beta})\left[1+\sum_{\lambda} \beta_{\lambda} Y_{\lambda 0}(\theta)\right],
$$

where $\boldsymbol{\beta} \equiv\left\{\beta_{\lambda}\right\}$ and $c_{v}(\boldsymbol{\beta})$ is a normalization factor to conserve the volume.

As the standard parameter set which corresponds to the actual nuclear potential, we employ the universal parameter set given in Table 1 of Ćwiok et al. [19] for the main results. Precisely speaking, we do not use the depth of the central potential as it is, but modify it for each nucleus in a way explained in Sec. [IC and Ref. [11]. We also use five other parameter sets, which we call Wahlborn [20], Rost 21], Chepurnov 22], Wyss-1 23], and Wyss-2 24] (see Table I of Ref. 25]), to confirm the independence of the conclusions from the choice of the standard parameter set.

Similarly to the Nilsson case, two constants $f_{a}$ and $f_{l s}$ are introduced by which to multiply the standard values of the surface diffuseness $a$ and the spin-orbit potential strength $\lambda_{\mathrm{SO}}$, respectively. By changing the combination of the values of these multipliers $\left(f_{a}, f_{l s}\right)$ from the standard values $(1,1)$, we examine how the prolate-shape dominance depends on the combined effects of the surface diffuseness and the spin-orbit potential.

It should be noted that the centrifugal potential enhances the effect of diffuseness on orbits having large angular momentum by pushing their wave functions out strongly on the surface. Thus, the effect of changing $a$ by several tens of percents is quite large despite the fact that the standard value $a=0.7 \mathrm{fm}$ is much smaller than the nuclear radius $\sim 10^{1} \mathrm{fm}$.

The multiplier $f_{l s}$ of the WS potential is approximately equivalent to $f_{l s}$ of the Nilsson potential because both of them are the multipliers to the spin-orbit potential. Indeed, as we will show in Sec. III] the same value of $f_{l s}$ seems to correspond to the same situation between the two kind of potentials as far as the ratio of prolate-shape nuclei concerns.

On the other hand, $f_{a}$ of the WS potential and $f_{l l}$ of the Nilsson potential work in different ways to change (literally or effectively) the radial profile of the potential. A finite-height cavity (i.e., square-well) potential is obtained in the limit $f_{a} \rightarrow 0$ of the WS potential and it can also be approximated by the Nilsson potential with a certain value of $f_{l l}$ (>1, dependent on the mass number $A$ ). A harmonic oscillator potential $\left(\propto r^{2}\right)$ is obtained by setting $f_{l l}=0$ of the Nilsson potential and it can also be approximately expressed by a certain value of $f_{a}$ ( $>1$, depending on $A$ ) for the WS potential. We will show in Sec. IIIA that $f_{a}=0,1$, and 2 correspond very roughly to $f_{l l}=1.5,1$, and 0 , respectively, as far as the ratio of prolate-shape nuclei concerns, which is an average over Oxygen to superheavy elements. One may also think roughly that WS potentials with $f_{a} \gtrsim 2$ cannot be expressed by Nilsson potentials while Nilsson potentials with $f_{l l} \gtrsim 1.5$ or $f_{l l}<0$ cannot be expressed by WS potentials.

In this paper, we calculate the ratio of prolate nuclei for $31 \times 19=589$ kinds of artificially modified WS potentials defined by the combination of values of the multipliers $\left(f_{l s}, f_{a}\right)$ in ranges $-1.5 \leq f_{l s} \leq 1.5$ and $0.2 \leq f_{a} \leq 2$ with $\Delta f_{l s}=\Delta f_{a}=0.1$.

Finally, we remark on the dependence of spin-orbit splittings, $\epsilon_{j=l-\frac{1}{2}}-\epsilon_{j=l+\frac{1}{2}}$, on the surface diffuseness. Concerning the Nilsson potential, the effects of two multipliers $f_{l s}$ and $f_{l l}$ are orthogonal and $f_{l l}$ never affects spin-orbit splittings. As for the WS potential, however, spin-orbit splittings can be affected by $f_{a}$ through the radial form factor $\nabla V_{\mathrm{WS}}$ in $V_{\mathrm{SO}}$ of Eq. (5). (All the WS parameter sets postulate $a_{\mathrm{SO}}=a_{\mathrm{CE}}$.) However, the net effect turns out to be small on the average after the integral is done over the radius.

In Fig. 1, we show the average value of spin-orbit splittings in ${ }^{208} \mathrm{~Pb}$ as a function of $f_{a}$. The potential is spherical and the parameter set is the universal set. In calculating the average, we consider all the pairs of levels 


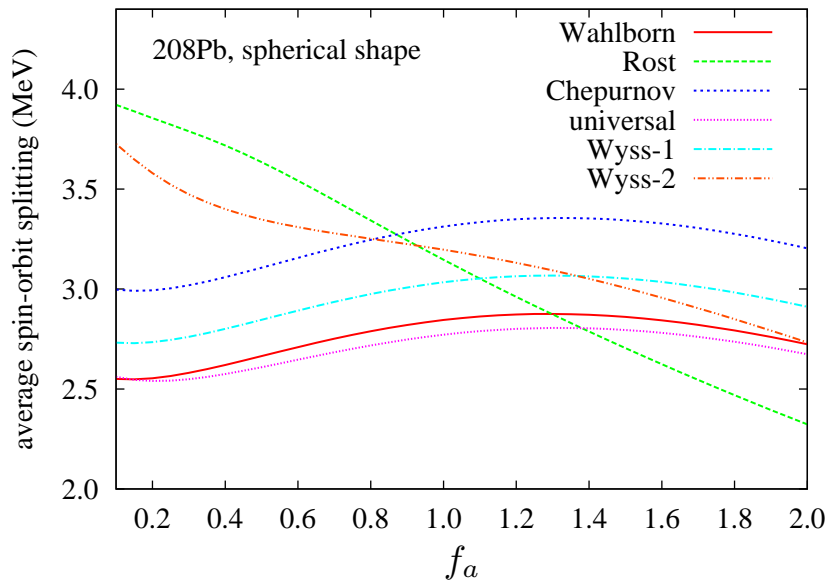

FIG. 1: Average spin-orbit splitting in units of $\mathrm{MeV}$ of ${ }^{208} \mathrm{~Pb}$ versus a multiplier $f_{a}$ to the surface diffuseness for six sets of parameters of the WS potential.

whose energies are negative throughout the plotted region $0.1 \leq f_{a} \leq 2$. The number of states in each pair of levels $(4 l+2)$ is used as the weight in the averaging procedure. We do not distinguish proton and neutron levels for this figure. For the Wahlborn, Chepurnov, universal, and Wyss-1 parameter sets, the variation in the average splitting is small $(\sim 10 \%)$. For the Rost and Wyss-2 parameter sets, the variation is larger (monotonically decreasing). We find that these different behaviors are originated in the difference between $R_{\mathrm{SO}}$ and $R_{\mathrm{CE}}$, i.e., the splitting does not change very much if $R_{\mathrm{SO}}=R_{\mathrm{CE}}$, while it is a monotonically decreasing (increasing) function of $f_{a}$ if $R_{\mathrm{SO}}<R_{\mathrm{CE}}\left(R_{\mathrm{SO}}>R_{\mathrm{CE}}\right)$. The spin-orbit splitting of each pair of levels (not shown) behaves in the same way as the average (shown) except when the upper level is close to zero energy.

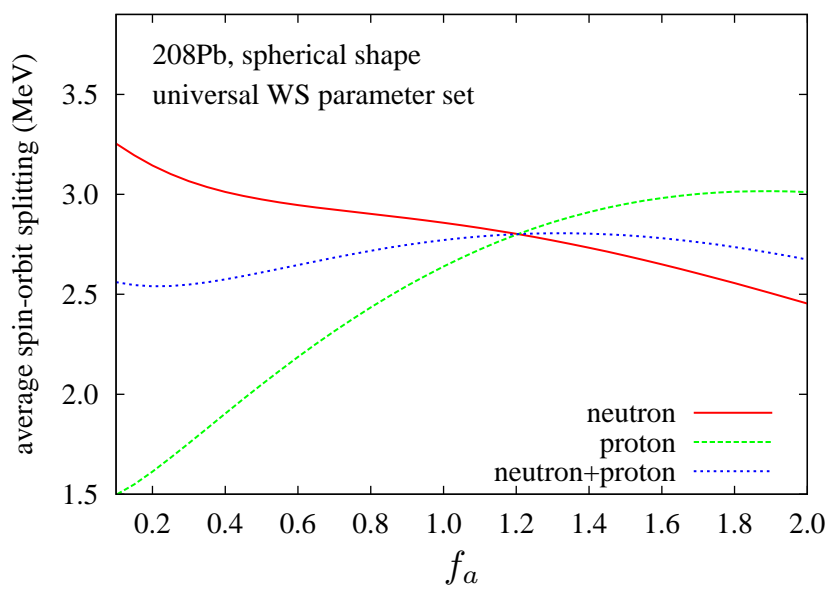

FIG. 2: Spin-orbit splittings in units of $\mathrm{MeV}$ of ${ }^{208} \mathrm{~Pb}$ averaged over neutron levels, proton levels, and all the levels versus $f_{a}$ calculated with the universal parameter set for the WS potential.
The universal parameter set is exceptional in the sense that $R_{\mathrm{SO}} / R_{\mathrm{CE}}<1$ for neutron and $>1$ for proton. We show the average over neutron levels and that over proton levels separately for the splittings calculated with this parameter set in Fig. 2. The curve labeled as "neutron+proton" is the same as the curve for this parameter set in Fig. 1. One can see that the neutron splitting decreases by $25 \%$ while the proton splitting increases by $100 \%$ in the plotted interval of $f_{a}$. It is not clear whether the effects cancel between neutrons' decreasing and and protons' increasing splittings concerning the quantities like the ratio of prolate nuclei. From this point of view, the universal parameter set may not be the most suitable set for the purpose of this paper. However, it is not a major demerit since the principal results of this paper like Fig. 3 and Fig. 15 do not look very different between any parameter sets. Anyway, we regard the universal parameter set as the standard and mainly show the results calculated with that parameter set.

\section{The Strutinsky method and its extensions}

The shape and energy of the ground state of each nucleus are determined by the shell correction method [2]. However, the conventional method cannot be applied to finite-depth potentials owing to the continuum part of the spectrum [26]. In order to cope with this difficulty, we employ an improved shell correction method which we have recently proposed (the reference density method [8]) based on the Kruppa prescription [9].

In shell-correction approaches, the total energy of a nucleus is assumed to be expressed as a sum of the macroscopic and microscopic parts,

$$
E=E_{\mathrm{mac}}+E_{\mathrm{mic}} \equiv E_{\mathrm{LDM}}+\left(E_{\mathrm{BCS}}-\tilde{E}_{\mathrm{BCS}}\right),
$$

where $E_{\mathrm{mac}}=E_{\mathrm{LDM}}$ is the energy of the liquid-drop model, whose parameters are taken from Ref. [27]. The energies, $E_{\mathrm{BCS}}$ and $\tilde{E}_{\mathrm{BCS}}$, are calculated as

$$
\left(\begin{array}{c}
E_{\mathrm{BCS}} \\
\tilde{E}_{\mathrm{BCS}}
\end{array}\right)=\int_{-\infty}^{+\infty}\left(\begin{array}{c}
v^{2}(\epsilon) g(\epsilon) \\
\tilde{v}^{2}(\epsilon) \tilde{g}(\epsilon)
\end{array}\right) \epsilon d \epsilon-\frac{1}{G}\left(\begin{array}{c}
\Delta^{2} \\
\tilde{\Delta}^{2}
\end{array}\right)
$$

where the occupation probability $v^{2}(\epsilon)$ and the pairing gap $\Delta\left(\tilde{v}^{2}(\epsilon)\right.$ and $\left.\tilde{\Delta}\right)$ are obtained by solving the BCS equation for the seniority-type pairing interaction, whose matrix elements are a constant $G$, with discrete level density $g(\epsilon)$ (Strutinsky smoothed level density $\tilde{g}(\epsilon)$ ).

The interaction strength $G$ is determined such that the smoothed pairing gap agrees with the empirical smooth trend,

$$
\tilde{\Delta}=f_{\Delta} \times 13 A^{-1 / 2}(\mathrm{MeV}),
$$

where the multiplier $f_{\Delta}$ is one (which corresponds to actual nuclei) for most of the calculations except in Sec. IIIB where we try three other values $\left(f_{\Delta}=0,0.7\right.$, $1.2)$. 
The depth of the central potential $V_{\mathrm{CE}}$ is adjusted for the consistency between the microscopic (in the ThomasFermi approximation) and macroscopic Fermi energies for spherical shapes neglecting the spin-orbit potential. This adjustment is necessary in order to treat nuclei far from the $\beta$ stability line because the available parameter sets of the WS potential do not reproduce the location of the drip lines predicted by the macroscopic part of energy. This adjustment is also indispensable when $f_{a} \neq$ 1 , because the Fermi level is strongly affected by $f_{a}$.

More details on the methods of calculations are described in Ref. [8].

\section{Numerical precision}

We have confirmed that numerically calculated singleparticle spectrum of the WS potentials is sufficiently precise to calculate the ratio of prolate nuclei. It should be noted that usually recommended size and frequency of the oscillator basis are not guaranteed to be sufficient to diagonalize Hamiltonians when potentials are artificially modified, i.e., when $f_{l s}$ or $f_{a}$ is not one.

We prepare the basis of diagonalization as eigenfunctions of a Hamiltonian of a nucleon of mass $m$ in a harmonic oscillator potential, whose frequency is determined in such a way that the major shell spacing is equal to $f_{\omega} \hbar \omega=f_{\omega} \times 41 A^{-1 / 3} \mathrm{MeV}$. Here, $f_{\omega}$ is an adjustment factor, usually takes on 1.2. When $f_{a}>1$, the spatial extension becomes larger so that the oscillator length of the basis $\propto f_{\omega}^{-1 / 2}$ should be increased, i.e., $f_{\omega}$ should be decreased. We determine empirically $f_{\omega}$ as a function of $f_{a}$,

$$
f_{\omega}= \begin{cases}1.2 & \left(0<f_{a} \leq 0.5\right) \\ 1.4-0.4 f_{a} & \left(0.5<f_{a} \leq 1.5\right) \\ 0.8 & \left(f_{a} \geq 1.5\right)\end{cases}
$$

so as to obtain more precise results with smaller basis.

The single-particle basis is truncated according to the maximum number of the oscillator quanta $N_{\text {osc }}$. In this paper, we diagonalize mostly in a subspace $N_{\text {osc }} \leq 20$. Concerning the calculations for Figs. 3, 4, 11, and 15, we consider only $N_{\text {osc }} \leq 16$ because of the required large computations. As we show in Sec. ПII the results are quite close to each other between the calculations with $N_{\text {osc }} \leq 16$ and $N_{\text {osc }} \leq 24$ as far as the ratio of prolate nuclei concerns.

\section{E. Set up of the numerical calculations}

For each potential specified by a set of multipliers $\left(f_{l s}, f_{l l}\right)$ or $\left(f_{l s}, f_{a}\right)$, we calculate the ground-state shape for all the even-even nuclei with $8 \leq Z \leq 126$ and $8 \leq N \leq 184$ between the proton and neutron drip lines predicted by the Bethe-Weizäcker (macroscopic) mass formula (2148 nuclei). To find the ground-state shape for each of these 2148 nuclei with each of the 589 kinds of the WS potentials, we calculate the nuclear total energy surface versus the quadrupole and hexadecapole deformation parameters $\left(\beta_{2}, \beta_{4}\right)$ at $51 \times 21=1071$ points in ranges $-0.5 \leq \beta_{2} \leq 0.5$ with $\Delta \beta_{2}=0.02$ and $-0.3 \leq \beta_{4} \leq 0.3$ with $\Delta \beta_{4}=0.03$. For the NilssonStrutinsky calculations of the 496 kinds of the Nilsson potentials, $\beta_{\lambda}$ should be read as $\epsilon_{\lambda}$.

The scale of required numerical computation is quite large. In order to obtain each of the six panels Fig. 3 (a) and Fig. 15 (a-e) corresponding to the different parameter sets of the WS potentials, we have to calculate the total energy $589 \times 2148 \times 1071=1.4 \times 10^{9}$ times, each time of which is for a nucleus using a given-shape potential. We have performed such computations in 64 threads in a PC cluster having eight Intel Core i7-920 CPU $(2.66 \mathrm{GHz}$, four physical = eight logical cores in a CPU). It has typically taken 28 hours for a physical core to complete a nuclear chart for a given combination of $\left(f_{l s}, f_{a}\right)$, and 20 days for the cluster to complete all the combinations, when we treat the WS potential in an oscillator space truncated by $N_{\text {osc }} \leq 16$. Compared with the WS potentials, it takes negligibly small time to perform the corresponding Nilsson-Strutinsky calculations.

\section{F. Definition of the ratio of prolate nuclei}

After completing all the Strutinsky-method calculations, we count the number of prolate, oblate, and spherical nuclei in each nuclear chart to calculate the ratio of prolate nuclei $R_{\mathrm{p}}$ as a measure of the prolate-shape dominance. We use two different definitions of the ratio in order to demonstrate that the main conclusions of this paper are not affected by the choice of the definition.

By $N_{\mathrm{o}}, N_{\mathrm{s}}$, and $N_{\mathrm{p}}$, let us denote the number of nuclei whose ground states are oblate $\left(\beta_{2} \leq-0.05\right)$, spherical $\left(\left|\beta_{2}\right|<0.05\right)$, and prolate $\left(\beta_{2} \geq 0.05\right)$, respectively, among the 2148 even-even nuclei defined in Sec.IIE, with those having positive Fermi levels excluded. Using these numbers, the ratio of prolate nuclei may be defined as

$$
R_{\mathrm{p}}^{\prime}=\frac{N_{\mathrm{p}}}{N_{\mathrm{p}}+N_{\mathrm{o}}}
$$

In Ref. [5], however, we have proposed a different definition in order to exclude shape transitional nuclei which often have an almost flat bottom in the nuclear energy curve ranging typically between $-0.2 \lesssim \beta_{2} \lesssim 0.2$. It is better to exclude them because precise ground states of such nuclei are superpositions of prolate and oblate shape states owing to large zero-point quantum fluctuations. There is little point whether the minimum happens to be located in the prolate or oblate side. After an extensive examination of the landscapes of total energy curves of all the even-even nuclei, we found that excluding spherical and transitional nuclei is nearly equivalent to including only those nuclei which have both prolate and oblate 
minima (discarding very shallow ones [5] ). Hence we have defined alternatively the ratio of prolate nuclei as

$$
R_{\mathrm{p}}=\frac{N_{\mathrm{p}}^{\mathrm{DM}}}{N_{\mathrm{p}}^{\text {DM }}+N_{\mathrm{o}}^{\text {DM }}}
$$

where $N_{\mathrm{p}}^{\text {DM }}\left(N_{\mathrm{o}}^{\text {DM }}\right)$ denotes the number of nuclei which has both oblate and prolate minima and the prolate (oblate) minima has a lower energy. (Superscript "DM" stands for "double-minimum").

In Sec. IIIA we will demonstrate that the main conclusions of this paper is not altered essentially whether we use either $R_{\mathrm{p}}$ or $R_{\mathrm{p}}^{\prime}$. The advantage of $R_{\mathrm{p}}$ is the exclusion of transitional nuclei while the merit of $R_{\mathrm{p}}^{\prime}$ is only simplicity. Hence we mainly use $R_{\mathrm{p}}$ in this paper.

\section{RESULTS}

\section{A. Ratio of prolate nuclei versus potential parameters}

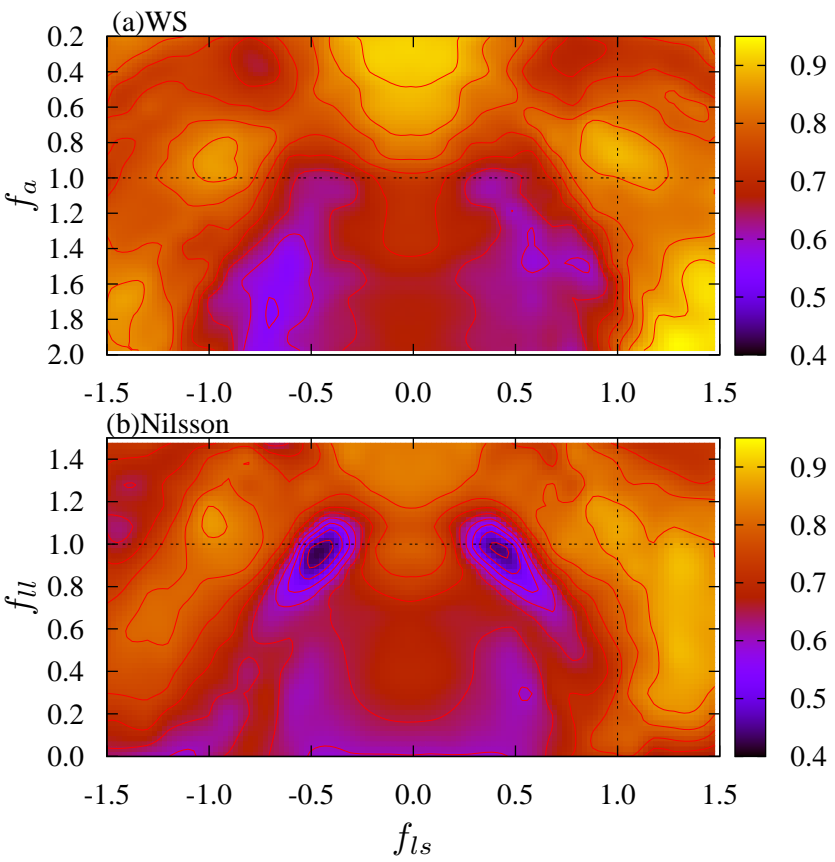

FIG. 3: The ratio of prolate nuclei $R_{\mathrm{p}}$ for the WS (a) and the Nilsson (b) potentials. The abscissa is the multiplier $f_{l s}$ to the spin-orbit potential for both panels. The ordinate is the multiplier $f_{a}$ to the surface diffuseness $a$ of the WS potential (a) and the multiplier $f_{l l}$ to the $\boldsymbol{l}^{2}$ potential of the Nilsson potential (b). The universal parameter set is used as the standard one for the WS potential.

In Fig. 3. the ratio of prolate nuclei $R_{\mathrm{p}}$ defined by Eq. (13) is shown as a function of $f_{l s}$ and $f_{a}$ for the WS potential (a) and $f_{l l}$ and $f_{l s}$ for the Nilsson potential (b). We use the universal parameter set [19] for the WS potential. Fourth order Legendre polynomials in $f_{l s}$ and

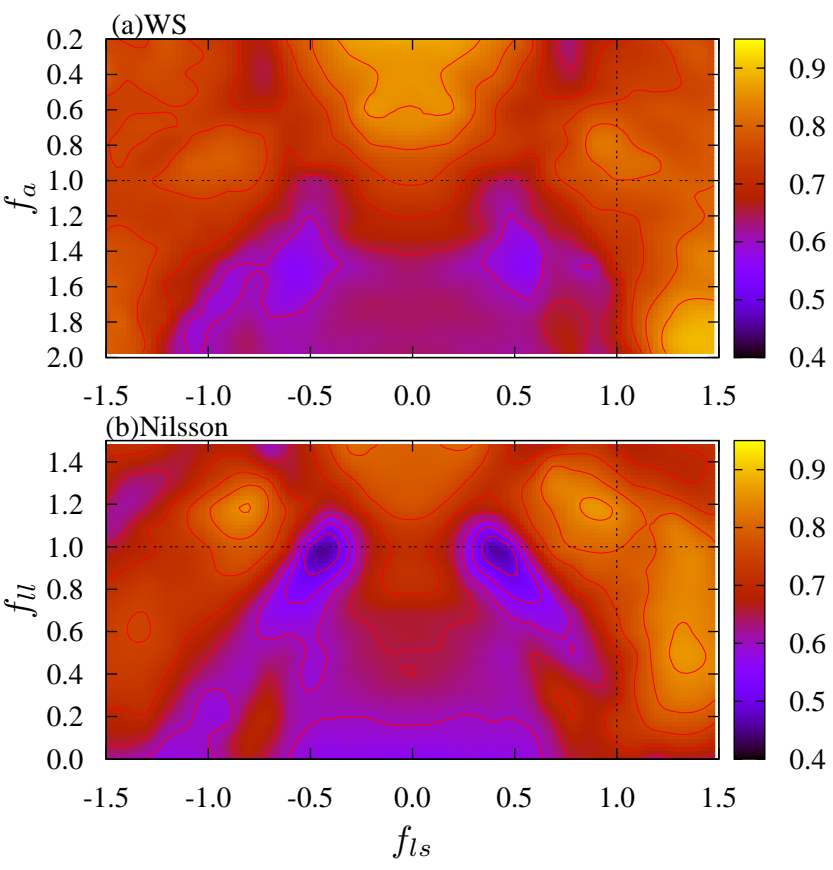

FIG. 4: Same as Fig. 3 but for a different definition of the ratio of prolate nuclei $R_{\mathrm{p}}^{\prime}$.

those in $f_{a}$ (or $f_{l l}$ ) are used for the interpolations to draw the contours.

In Fig. 4, the ratio of prolate nuclei $R_{\mathrm{p}}^{\prime}$ of a simpler definition of Eq. (12) is shown in the same manner as $R_{\mathrm{p}}$ in Fig. 3. One can see the behavior of $R_{\mathrm{p}}^{\prime}$ is quite similar to that of $R_{\mathrm{p}}$. It can also be confirmed in detail in Figs. 50 8, which show curves for $R_{\mathrm{p}}$ and $R_{\mathrm{p}}^{\prime}$ in various cross sections of the panels in Figs. 3 and 4 . The distance between the two curves is mostly $\sim 0.05$. The largest discrepancy is 0.11 at $\left(f_{l s}, f_{l l}\right)=(0,1)$ in Fig. 6(b). We have also checked that a third definition

$$
R_{\mathrm{p}}^{\prime \prime}=\frac{N_{\mathrm{p}}}{N_{\mathrm{p}}+N_{\mathrm{s}}+N_{\mathrm{o}}}
$$

does not change the behavior very much, either (no figures). Therefore, the following discussions are independent of the definition of the ratio of prolate nuclei.

Result for the Nilsson potential shown in Fig. 3(b) is essentially the same as Fig. 1 of Ref. [5], except that the area of $f_{l l}<0$ is not shown in this paper.

In a line $f_{l s}=0$ (Fig. $8(\mathrm{a})$ ), i.e., when there is no spin-orbit potential, $R_{\mathrm{p}}\left(R_{\mathrm{p}}^{\prime}\right)$ increases monotonically as a function of $f_{l l}$, from $60 \%(56 \%)$ at $f_{l l}=0$ (i.e., the harmonic oscillator potential) to $80 \%(71 \%)$ at $f_{l l}=1$ (corresponding to the actual surface thickness). This behavior agrees with Frisk's argument [3] that a cavity(like) potential prefers prolate shapes more than oblate ones.

In a line $f_{l l}=1$ (Fig. 6(b)), i.e., with the surface diffuseness of actual nuclei, $R_{\mathrm{p}}$ as well as $R_{\mathrm{p}}^{\prime}$ oscillates strongly as a function of $f_{l s}$. It takes local maximum values at $f_{l s} \simeq \pm 1$ and local minimum values at $f_{l s} \simeq \pm 0.5$. 

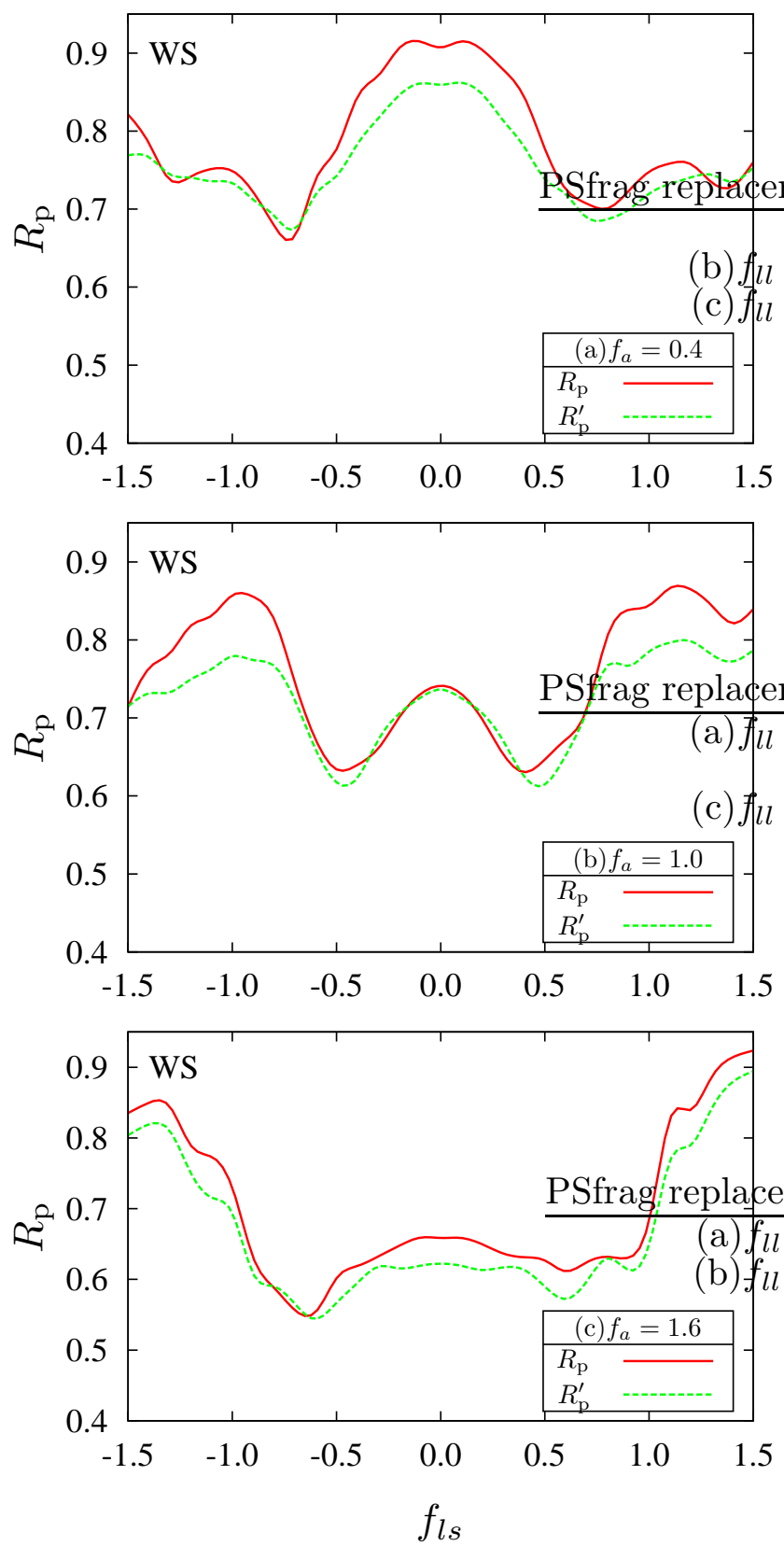

FIG. 5: The ratio of prolate nuclei $R_{\mathrm{p}}$ and $R_{\mathrm{p}}^{\prime}$ for the WS potential for (a) $f_{a}=0.4$, (b) $f_{a}=1$, and (c) $f_{a}=1.6$ versus the multiplier to the spin-orbit strength $f_{l s}$.

It means a strong interference between the effects of the $l s$ and $\boldsymbol{l}^{2}$ potentials. Minima of $R_{\mathrm{p}}$ are located close to this line $f_{l l}=1$ where $R_{\mathrm{p}} \simeq 40 \%$, i.e., oblate shapes dominate over prolate shapes if the spin-orbit potential is weakened by $50 \%$ while keeping the surface diffuseness of the potentials at the standard value.

The result for the WS potential is shown in Fig. 3(a). 31] It looks quite similar to Fig. 3(b) for the Nilsson potential.

In a line $f_{l s}=0$ (Fig. $\left.7(\mathrm{a})\right), R_{\mathrm{p}}\left(R_{\mathrm{p}}^{\prime}\right)$ increases almost monotonically as a function of $-f_{a}$, from $68 \%(64 \%)$ at
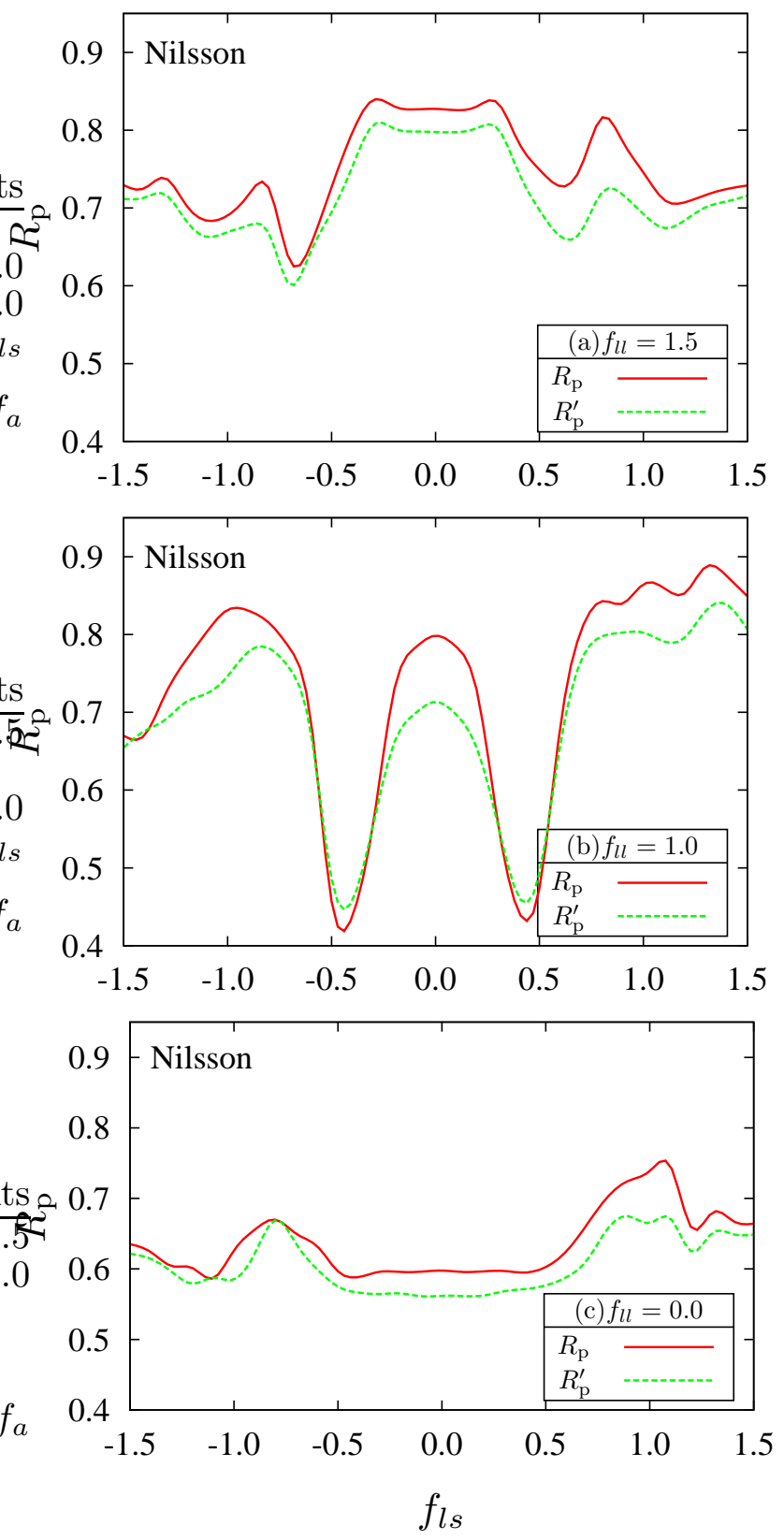

FIG. 6: The ratio of prolate nuclei $R_{\mathrm{p}}$ and $R_{\mathrm{p}}^{\prime}$ for the Nilsson potential for (a) $f_{l l}=1.5$, (b) $f_{l l}=1$, and (c) $f_{l l}=0$ versus the multiplier to the spin-orbit strength $f_{l s}$.

$f_{a}=2$ to $91 \%(86 \%)$ at $f_{a}=0.2$. This behavior during the transition from the harmonic oscillator to a cavitylike potentials agrees qualitatively with that of the Nilsson potential and with Frisk's argument [3]. Quantitatively, however, $R_{\mathrm{p}}=R_{\mathrm{p}}^{\prime}=73 \%$ at $f_{a}=1$ (actual nuclear central potential) is not sufficiently dominant. The situation is worse in a line $f_{l s}=0.5$ (Fig. 7 (b)). Only by the assist of the actual-strength spin-orbit potential, i.e., $f_{l s}=1$ (Fig. $\left.7(\mathrm{c})\right), R_{\mathrm{p}}$ can exceed $80 \%$ when $f_{a}=1$.

As for the Nilsson potential, this assist of the spin-orbit potential works stronger to change the landscape of the 

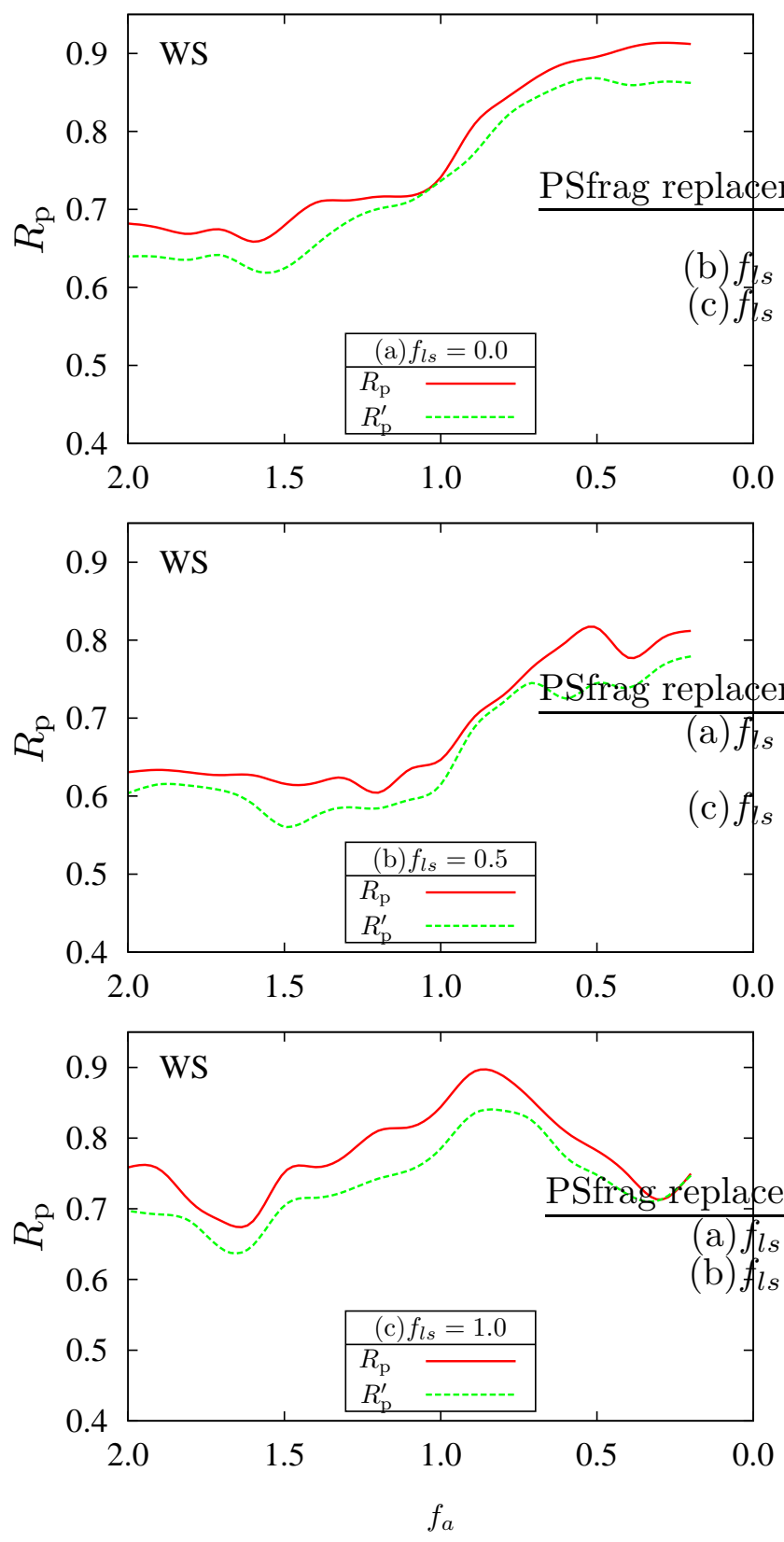

FIG. 7: The ratio of prolate nuclei $R_{\mathrm{p}}$ and $R_{\mathrm{p}}^{\prime}$ for the WS potential for (a) $f_{l s}=0$, (b) $f_{l s}=0.5$, and (c) $f_{l s}=1$ versus the multiplier to the surface diffuseness $f_{a}$.

curve. When $f_{l s}=0.5$, it generates a large-amplitude oscillation versus $f_{l l}$ (Fig. 8(b)), whose minimum at $f_{l l}=$ 0.95 corresponds to the minimum at $\left(f_{l s}, f_{l l}\right)=(0.45,1)$ in Fig. 6(b). It is this oscillation that has moved two local minima upward in two-parameter plots of $R_{\mathrm{p}}$ and of $R_{\mathrm{p}}^{\prime}$ compared with those for the WS potential: For the WS potential (Fig. 3 (a)), the minima are located around a line $f_{a} \simeq 1.6$, not $f_{a}=1$, while for the Nilsson potential (Fig. 3(b)), they are just in a line $f_{l l}=1$. The amplitude of the oscillation in a line $f_{l l}=1$ (Fig. [6(b)) is larger than that in a line $f_{a}=1.6$ (Fig. 囵(c)) or that in a line
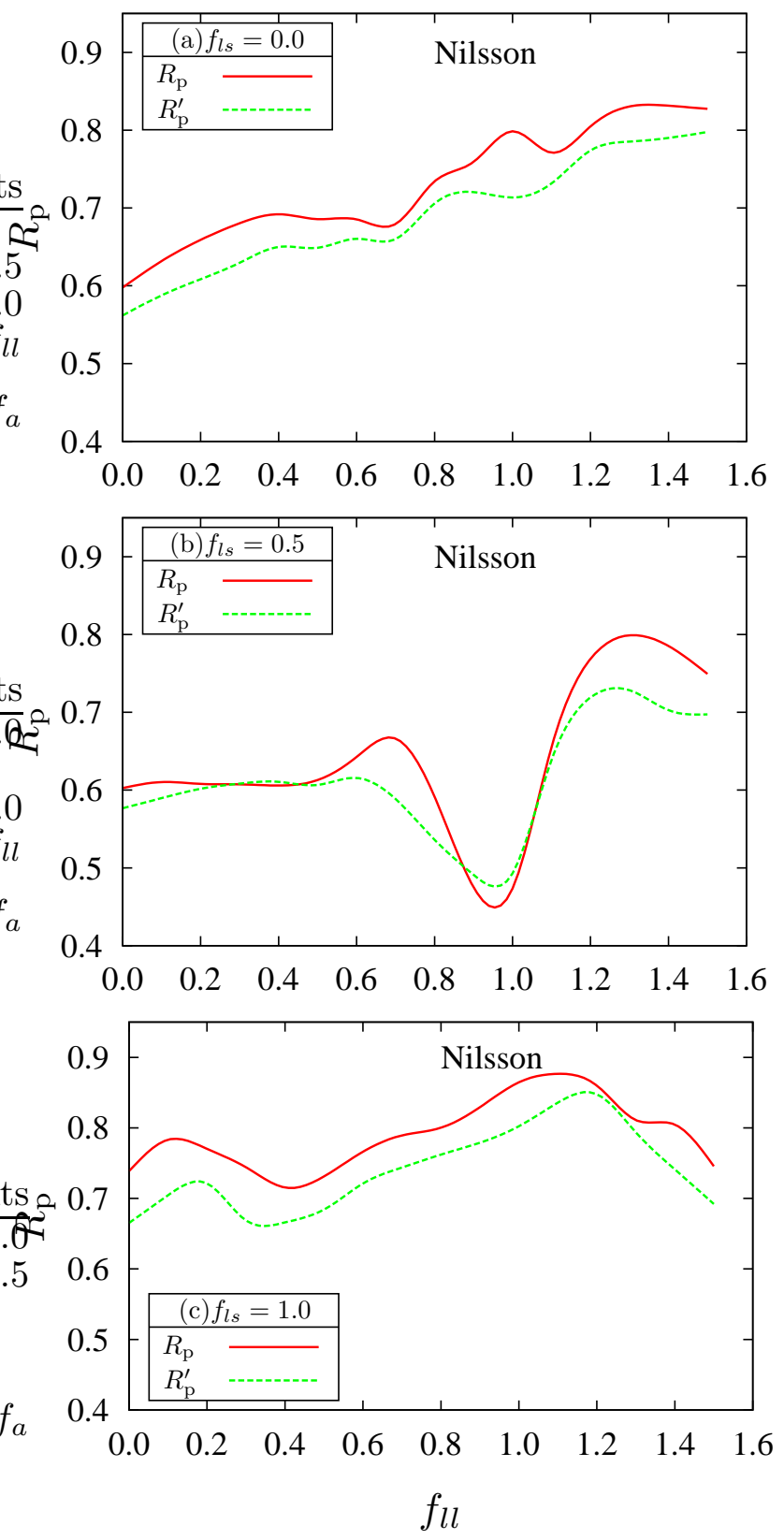

FIG. 8: The ratio of prolate nuclei $R_{\mathrm{p}}$ and $R_{\mathrm{p}}^{\prime}$ for the Nilsson potential for (a) $f_{l s}=0$, (b) $f_{l s}=0.5$, and (c) $f_{l s}=1$ versus the multiplier to the $\boldsymbol{l}^{2}$ term $f_{l l}$.

$f_{a}=1$ (Fig. 5 (b)) by a factor $\simeq 3$. The reason for the exaggeration of the amplitude of the oscillation by the Nilsson potential may be the approximate treatment of the radial profile of the potential in terms of the $l^{2}$ term and the affinity between this $l^{2}$ term and the $l s$ term in a sense that both of them contain the same orbital angular momentum operator $\boldsymbol{l}$. Anyway, we have confirmed a fact that the interference also exists for the WS potential.

In Fig. 9, we show that our single-particle basis is suffi-

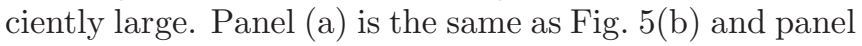
(b) is the same as Fig. [7(a) except that results with three 

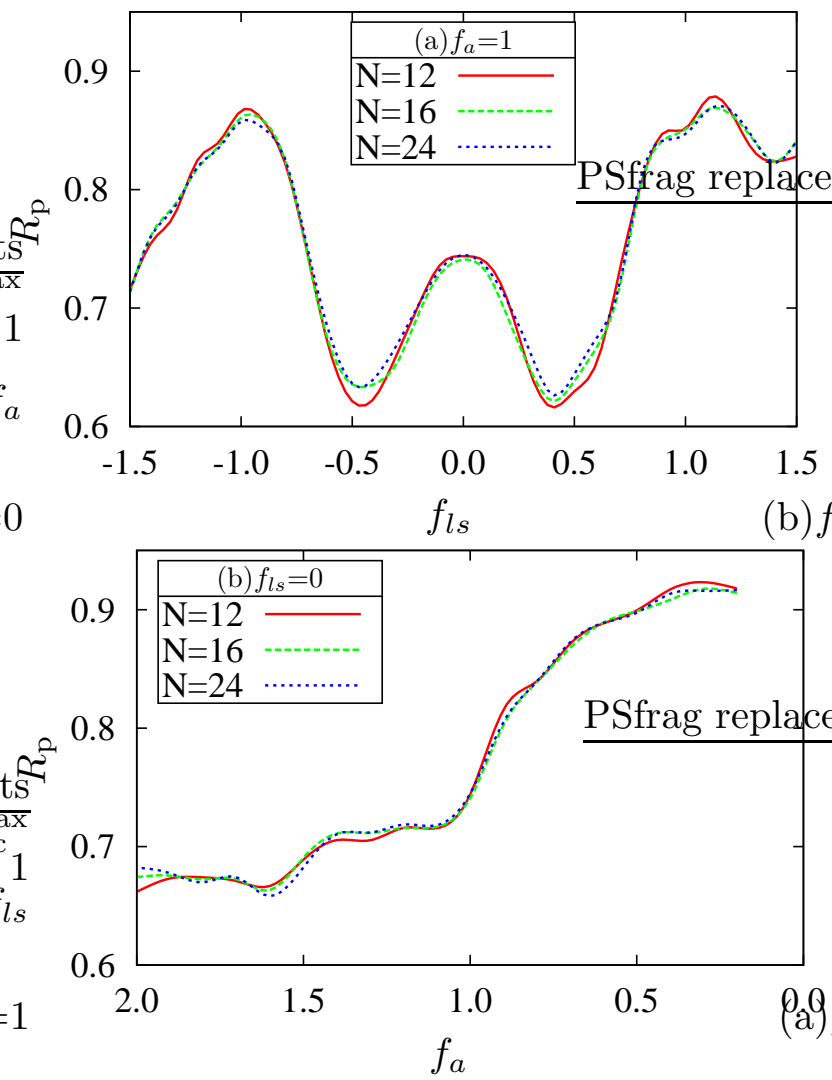

FIG. 9: Dependence of the ratio of prolate nuclei $R_{\mathrm{p}}$ on the size of the oscillator basis specified by $N_{\text {osc }}$, which is the maximum oscillator quantum number of the single-particle basis. In panel (a), $R_{\mathrm{p}}$ is plotted versus the multiplier to the spinorbit potential $f_{l s}$ while the surface diffuseness is frozen at the standard value $\left(f_{a}=1\right)$. In panel $(\mathrm{b}), R_{\mathrm{p}}$ is plotted versus the multiplier to the surface diffuseness $f_{a}$ while the spin-orbit potential is kept turned off $\left(f_{l s}=0\right)$.

different sizes of basis are compared. One can see in both panels that the curve for $N_{\text {osc }} \leq 16$ is quite close to the curve for $N_{\text {osc }} \leq 24$.

\section{B. The effect of the pairing strength}

In Fig. 10, the ratio of prolate nuclei $R_{\mathrm{p}}$ is shown for four values of the multiplier $f_{\Delta}$ appearing in Eq. (10) for the WS potential. The pairing strength can be controlled with this multiplier.

In panel (a), $R_{\mathrm{p}}$ is shown as a function of $f_{l s}$ while the surface diffuseness is the standard one $\left(f_{a}=1\right)$. The short dash curve labeled as $f_{\Delta}=1.0$ is the same as the solid curve in Fig. 5(b). One can see that a stronger pairing increases the amplitude of oscillation. The same tendency has also been found for the Nilsson potential [11]. The change is largest at prolate-shape dominant points of $f_{l s} \sim \pm 1$, where $R_{\mathrm{p}}$ is increased further from a high value to a higher value. This can be understood that generally shallower minima in oblate nuclei are more easily
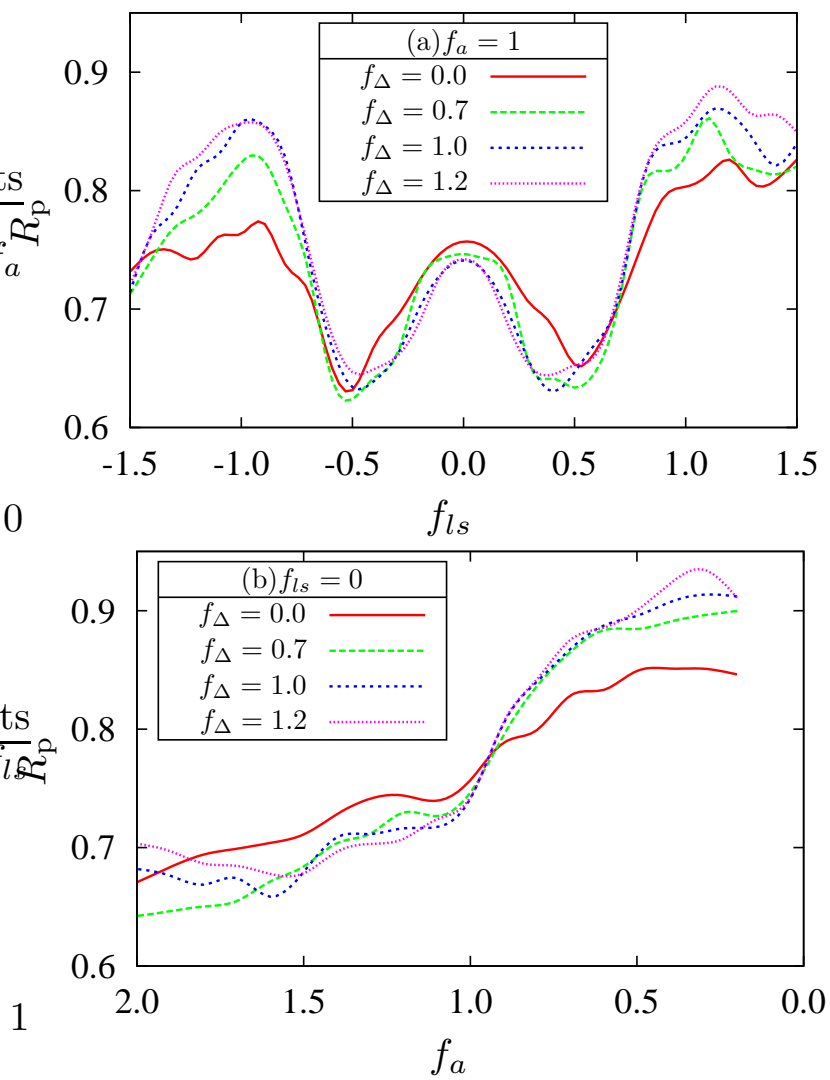

FIG. 10: The dependence of the ratio of prolate nuclei $R_{\mathrm{p}}$ on the pairing strength for the WS potential. Four kinds of curves correspond to different values of a modification factor $f_{\Delta}$ which determines the strength of the paring force. For $f_{\Delta}=1$, the empirical trend is used. For $f_{\Delta}=0$, there is no pairing correlation. Panel (a): as a function of the spin-orbit strength $f_{l s}$ with a normal diffuseness $f_{a}=1$. Panel (b): as a function of the diffuseness $f_{a}$ without a spin-orbit potential.

dissolved and merged with the other minima to form a single spherical minimum than generally deeper minima in prolate nuclei.

In panel (b), $R_{\mathrm{p}}$ is drawn versus $f_{a}$ while the spin-orbit potential is kept turned off $\left(f_{l s}=0\right)$. The short dash curve labeled as $f_{\Delta}=1.0$ is the same as the solid curve in Fig. 7(a). One can see an overall trend that stronger pairing correlation increases the magnitude of the slope. Pairing correlation increases $R_{\mathrm{p}}$ at $f_{a} \lesssim 1$, which may be understood by the same interpretation given in the last paragraph. Pairing correlation decreases $R_{\mathrm{p}}$ at $1 \lesssim f_{a} \lesssim$ 1.5. At $f_{a} \gtrsim 1.5$, curves are reordered and the trend of monotonic increase of $R_{\mathrm{p}}$ versus $-f_{a}$ is reversed for $f_{\Delta} \geq$ 1. These behaviors require a different interpretation yet to be explored.

\section{Mass dependence of the ratio of prolate nuclei}

We decompose Fig. 3(a) into three mass regions, $A \leq$ $100,100<A \leq 200$, and $A>200$ to obtain the three 

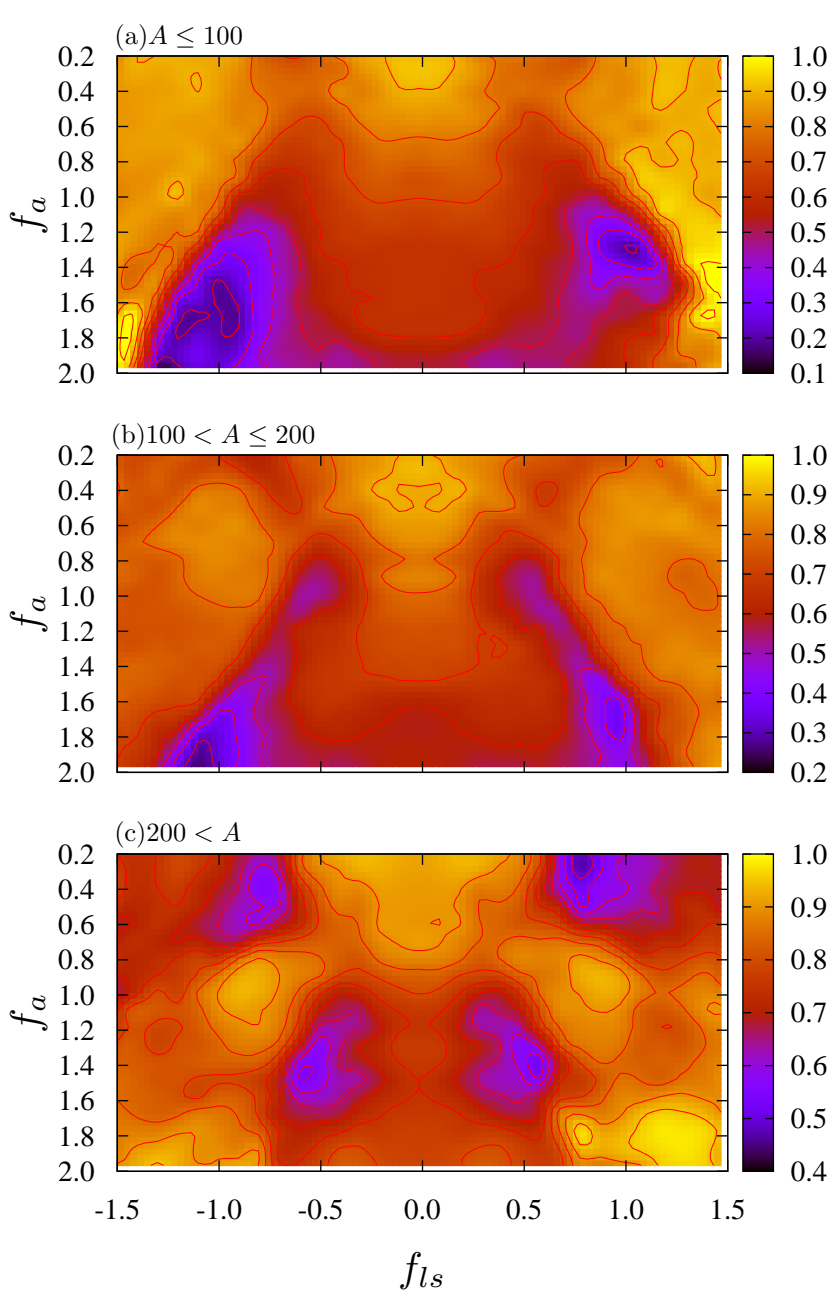

FIG. 11: Same as in Fig. 3(a) but decomposed into a light $A \leq 100$ (a), a medium $100<A \leq 200$ (b), and a heavy $200<A$ (c) mass regions.

panels of Fig. 11. One can see that the patterns in these three mass regions are rather different; the numbers of oscillations in both directions $\left(f_{a}\right.$ and $\left.f_{l s}\right)$ are larger in the heavier mass region. Namely, the ratio of prolate nuclei changes more rapidly when the potential is modified. This pattern change can be understood by the fact that the level density is larger in heavier system, where the prolate- and oblate-driving orbits coexist in a narrow energy range at the Fermi surface and a modification of the potential more easily changes the equilibrium deformation. Alternatively, one can think that the pattern is "shrunken" in two axes from the light to the heavy regions, although new pronounced low $R_{\mathrm{p}}$ areas appear in the range of small $f_{a} \lesssim 0.5$ in the heavy mass region (Fig. 11 (c)). The shrinkage in the $f_{a}$ axis may be ascribed to the ratio of a constant thickness $a$ to the nuclear radius $\propto A^{1 / 3}$, while that in the $f_{l s}$ axis may be related to the ratio of the spin-orbit splitting $\propto N_{\text {osc }} \propto A^{1 / 3}$ to the major shell spacing $\propto A^{-1 / 3}$. This shrinkage may cause a blurring of the pattern averaged over all the mass re- gions. It may be possible that, by employing a properly $A$-scaled parametrizations for the surface diffuseness $a$ and the spin-orbit potential strength $\lambda_{\mathrm{so}}$, instead of simple constant multipliers $f_{a}$ and $f_{l s}$, one obtains a little sharper pattern than Fig. 3 .

\section{Absolute number of nuclei of prolate, oblate, and spherical shapes}

In Fig. 12 we show not ratios but absolute numbers of nuclei of prolate, oblate, and spherical shapes at their ground states, i.e., $N_{\mathrm{p}}, N_{\mathrm{o}}$, and $N_{\mathrm{s}}$ defined in Sec. 【F The numbers of nuclei are plotted versus $f_{l s}$ while $f_{a}$ is frozen at 1 . Because these numbers depend on the mass number more strongly than ratios, we discuss separately the light, medium, and heavy mass regions introduced in Sec. IIIC, As it is discussed in the previous subsection, one observes considerable differences in three mass regions; the three numbers, $N_{\mathrm{p}}, N_{\mathrm{o}}$, and $N_{\mathrm{s}}$, oscillate more frequently in the heavy mass region. In Fig. 12(a), which shows the numbers of nuclei in the light mass region, the oscillation in $N_{\mathrm{p}}$ for $\left|f_{l s}\right| \leq 0.8$ can be ascribed to shape transitions of the ground state of each nuclei between prolate and spherical shapes because $N_{\mathrm{o}}$ is almost constant in that interval of $f_{l s}$. In Fig. 12(b), which is for the medium mass region, the amplitude of oscillation in $N_{\mathrm{p}}$ is most pronounced among the three mass regions. The oscillation is attributed evenly to shape transitions between prolate and spherical shapes and those between prolate and oblate shapes. In Fig. 12(c), which corresponds to the heavy mass region, the amplitude of the oscillation in $N_{\mathrm{p}}$ is reduced. This may be a consequence of the fact that, from the light to the heavy regions, the prolate nuclei continues to become more and more dominant over spherical and oblate nuclei for the normal diffuseness $f_{a}=1$.

\section{E. Map of deformation on the nuclear chart}

In Fig. 13 we plot the quadrupole deformation parameter $\beta_{2}$ of the ground states of even-even nuclei in the $(N, Z)$ plane. In panels $(\mathrm{a}-\mathrm{c})$, the WS potential is used. The parameter sets are the universal parameter set which is the choice of this paper (a), the Chepurnov parameter set 22] which is one of the classical (b), and the Wyss-2 24] parameter set which is one of the latest (c). In panel (d), the Nilsson potential is used and $\epsilon_{2}$ is plotted instead of $\beta_{2}$. Comparing the predictions of these potential parameter sets, one sees that the locations of prolate and oblate nuclei are roughly the same among the four panels. This means that the conclusions of this paper is not restricted to the mainly employed universal parameter set.

With a more careful look at these maps, one finds that a rectangular area $28<Z \lesssim 40,50<N<82$ is the area where the largest number of nuclei have differently 

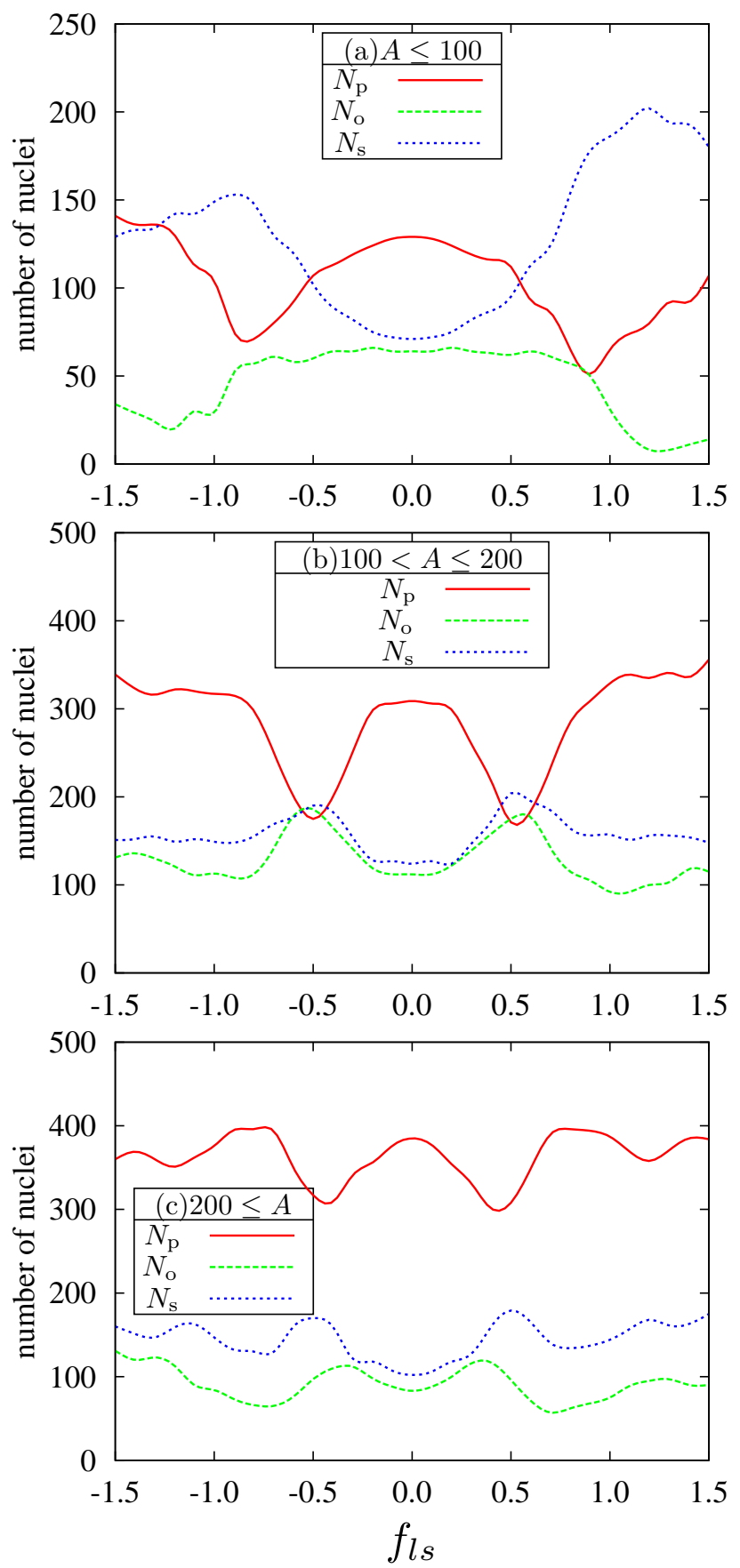

FIG. 12: The number of prolate $\left(N_{\mathrm{p}}\right)$, oblate $\left(N_{\mathrm{o}}\right)$, and spherical nuclei $\left(N_{\mathrm{s}}\right)$ in a light (a), a medium (b), and a heavy (c) mass regions as a function of $f_{l s}$ (with $f_{a}=1$ ).

predicted shapes by different parameter sets; the prediction of the Nilsson potential is also very different from those of the WS potentials. In this area, nuclei of large prolate deformations and those of large oblate deformations are located side-by-side directly, meaning the first order phase transition in the shape. A similar situation that the distinct prolate and oblate minima coexist in the same energy region has been extensively discussed in the $A \sim 80$ region, see e.g. Ref. 28]. Compared to the $A \sim 80$ region, this rectangular area is much wider so that it may be a more suitable region to study the issue of the shape coexistence and/or the shape phase transition in the light of recent developments of unstable beam facilities. It should be noticed that a smaller area $28<Z, N \lesssim 40$ has a similar characteristic, which provides typical examples of the shape coexistence phenomenon [29], where the spherical shape also play an important role.

The deformation map changes drastically when the multipliers are changed. In Fig. 14, we plot the quadrupole deformation $\beta_{2}$ predicted with the universal parameter set for the WS potential modified with a multiplier $f_{l s}=0.5$ (a), 0 (b) , -0.5 (c), and -1 (d) while $f_{a}$ is frozen at 1 . Comparing these four panels together with Fig. 13(a) for $f_{l s}=1$, one sees a substantial change of the distribution of the shapes caused by varying the value of $f_{l s}$.

One also notices that positive and negative values of $f_{l s}$ of the same magnitude (i.e., Fig. 13(a) and Fig. 14(d), Fig. 14(a) and Fig. 14(c)) lead to very similar distributions.

For $f_{l s}= \pm 1$, oblate-shape nuclei are located in a narrow region just below major-shell closures. On the other hand, for $f_{l s}= \pm 0.5$, they occupy a large area in the latter half of a major-shell filling. The largest of such areas are in $50 \leq Z \leq 80,90 \leq N \leq 120$ in Fig. 14(a,c).

It is interesting that parameters $f_{l s}=0$ and $f_{l s}= \pm 1$, which lead to similar levels of prolate-shape dominance, have completely different shape distribution maps. This seems mainly because, when the spin-orbit potential is missing $\left(f_{l s}=0\right)$, subshell closures are strong enough to subdivide conventional major shells.

\section{F. Dependence on WS parameters}

In Fig. 15, we show the ratio of prolate nuclei $R_{\mathrm{p}}$ for various WS parameter sets. Combined with Fig. 3 (a), all the six sets of the WS potentials are investigated. In all the figures the basic pattern of the contour plot is the same, although the precise positions of the low $R_{\mathrm{p}}$ regions are slightly shifted in each case. Especially, the physical points, $f_{l s}=f_{a}=1$, is near one of the highest points, indicating the strong prolate-shape dominance for all parameter sets. By comparing them, one can say that the conclusions of this paper are not essentially altered by changing the standard potential.

\section{G. Single-particle level densities}

We give an example to illustrate the way how the potential parameter changes the shape of a nucleus through the change of the level density. We choose a nucleus ${ }_{68}^{172} \mathrm{Er}_{104}$, which is located near the center of a region $52 \leq Z \leq 80,90 \leq N \leq 110$, where the ground state 

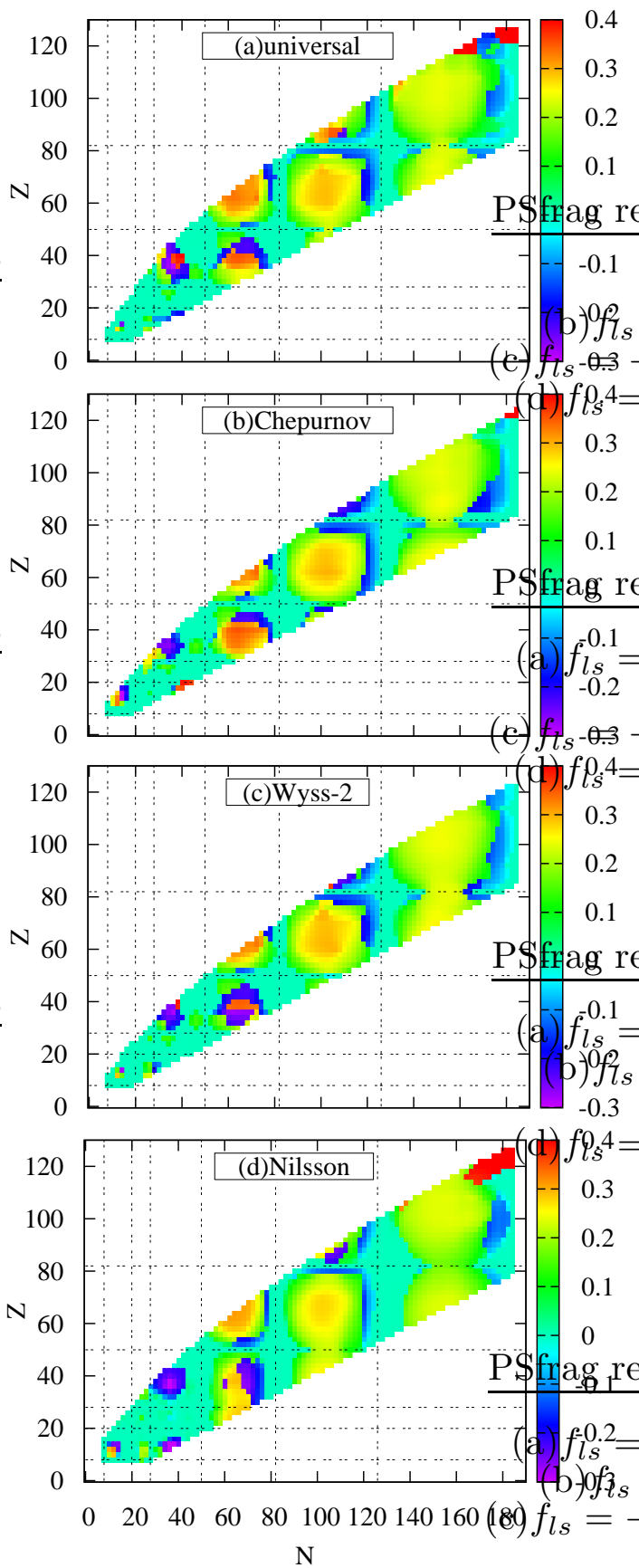

FIG. 13: Quadrupole deformation parameter $\beta_{2}$ of the ground states of even-even nuclei calculated with standard parameters for the WS potential $(\mathrm{a}-\mathrm{c})$ and the Nilsson potential $(\mathrm{d})$. The used parameter set for the WS potential are the universal (a), the Chepurnov (b), and the Wyss-2 (c).

is prolate for the standard potential and oblate for a modified potential with $\left(f_{l s}, f_{a}\right)=(0.5,1)$ as shown in Fig. 14(a).

In Fig. [16] the oscillating part of the neutron's level density defined by,

$$
\delta \tilde{g}(\epsilon)=\tilde{g}_{\gamma=0.5}(\epsilon)-\tilde{g}_{\gamma=1.2}(\epsilon),
$$

of the ground state of this nucleus is plotted as a func-
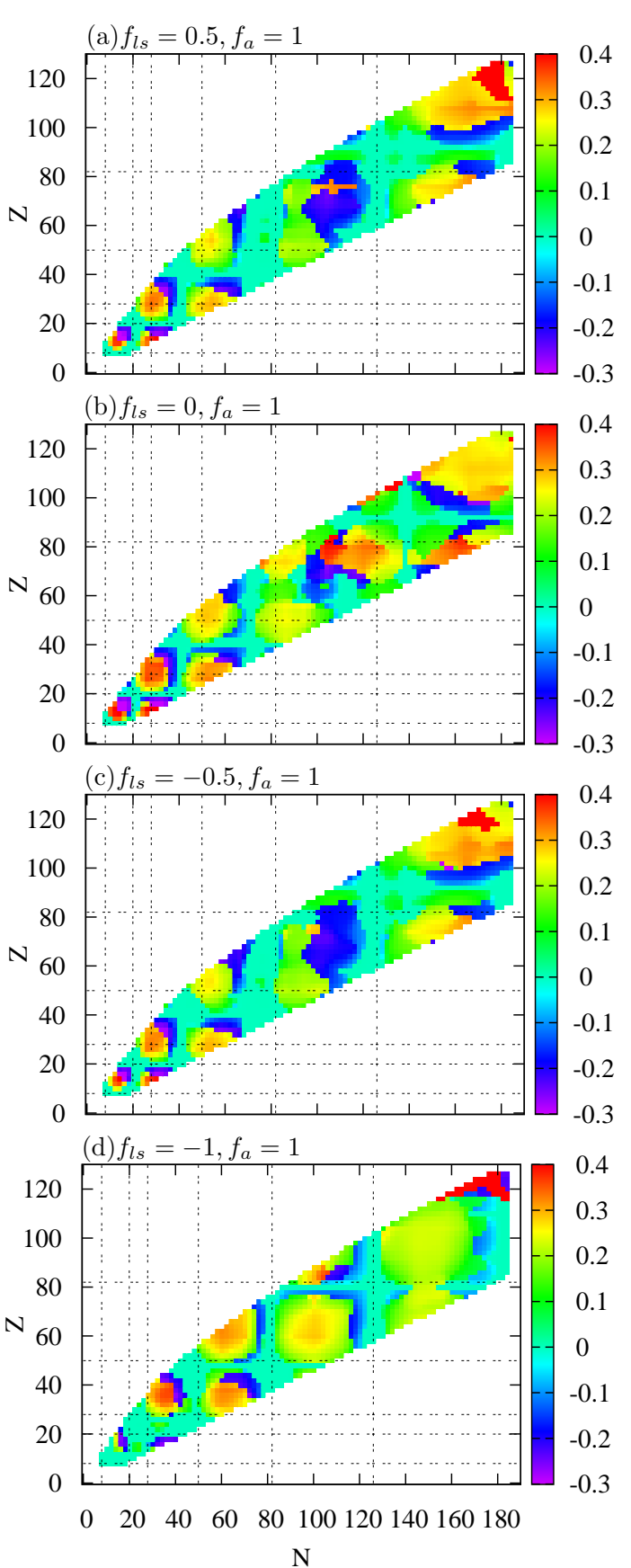

FIG. 14: Quadrupole deformation parameter $\beta_{2}$ of the ground states of even-even nuclei using the universal parameter set for the WS potential with multipliers $f_{a}=1$ and $f_{l s}=0.5$ (a), 0 (b), -0.5 (c), and -1 (d). Horizontal and vertical dot lines indicate the magic numbers of actual nuclei, not of the modified potentials.

tion of the multiplier to the spin-orbit potential $f_{l s}$ (abscissa) and the single particle energy in unit of $\hbar \omega$ (ordinate). The Fermi level for neutrons is designated with a black curve. The quantity $\gamma$ in Eq. (15) is the Strutinsky smoothing parameter in unit of $\hbar \omega$, and the value $\gamma=0.5$ is chosen to clearly show the oscillation due to the shell effect relative to the average level density with 


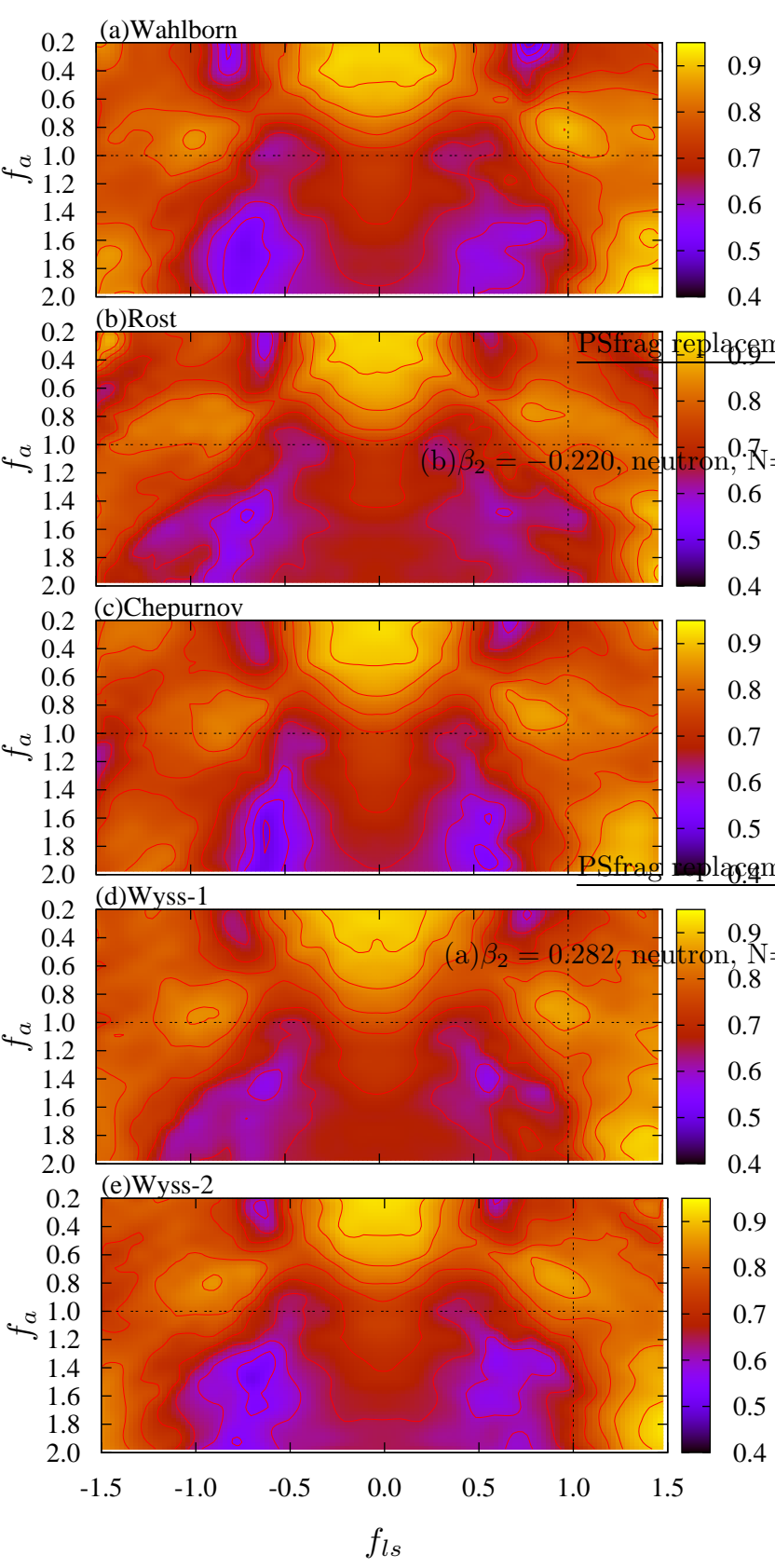

FIG. 15: Ratio of prolate nuclei $R_{\mathrm{p}}$ calculated by using different WS parameter sets as the standard, the Wahlborn (a), the Rost (b), the Chepurnov (c), Wyss-1 (d), and Wyss-2 (e).

$\gamma=1.2$, with which all Strutinsky smoothed quantities are calculated.

Fig. 16(a) shows the level density at a prolate shape $\left(\beta_{2}=0.282\right)$, in which the level density at the Fermi level is low (high) at $f_{l s}=1(0.5)$. Fig. 16(b) shows the level density at an oblate shape $\left(\beta_{2}=-0.220\right)$, in which the level density at the Fermi level is high (low) at $f_{l s}=1$ (0.5). The change from a prolate to an oblate shape of this nucleus due to the change of $f_{l s}$ from 1 to 0.5 can be explained in this way in the macroscopic-microscopic
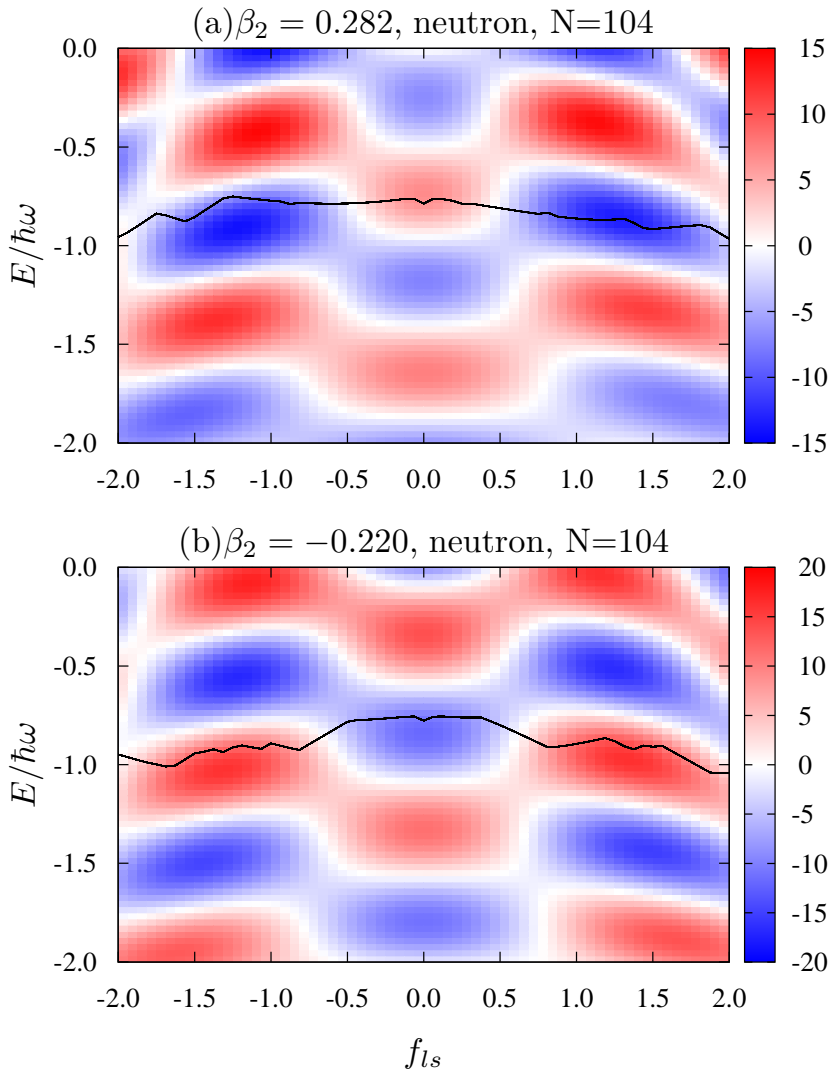

FIG. 16: Oscillating part of the neutron's single-particle level density $\delta \tilde{g}(\epsilon)$ of ${ }^{172} \mathrm{Er}$ as a function of the multiplier to the spin-orbit potential $f_{l s}$ and the single-particle energy divided by $\hbar \omega$. The shape of the potential is prolate in panel (a) and oblate in panel (b). The Fermi level is designated with a black curve.

theory.

\section{H. Prolate, oblate, and spherical ratios as functions of Fermi energy}

As it is explained in detail in Sec. IIE we have calculated the ground state masses of 2148 even-even nuclei in the nuclear chart for each modified set of parameters of the WS potential. In view of the results, there are many interesting findings apart from the prolate-shape dominance. Among them, we present the result on how the deformation of unstable nuclei changes compared with the stable nuclei. In Fig. 17 three ratios of the prolate, oblate, and spherical nuclei, i.e., $R_{\mathrm{p}}^{\prime \prime}$ defined by Eq. (14) and

$$
R_{\mathrm{o}}^{\prime \prime}=\frac{N_{\mathrm{o}}}{N_{\mathrm{p}}+N_{\mathrm{s}}+N_{\mathrm{o}}}, \quad R_{\mathrm{s}}^{\prime \prime}=\frac{N_{\mathrm{s}}}{N_{\mathrm{p}}+N_{\mathrm{s}}+N_{\mathrm{o}}}
$$

are shown as functions of the neutron (panel (a)) or proton (panel (b)) Fermi energy. Note that these ratios are now defined with including $N_{\mathrm{s}}$ in contrast to the definitions of $R_{\mathrm{p}}$ and $R_{\mathrm{p}}^{\prime}$ used in previous subsections which 

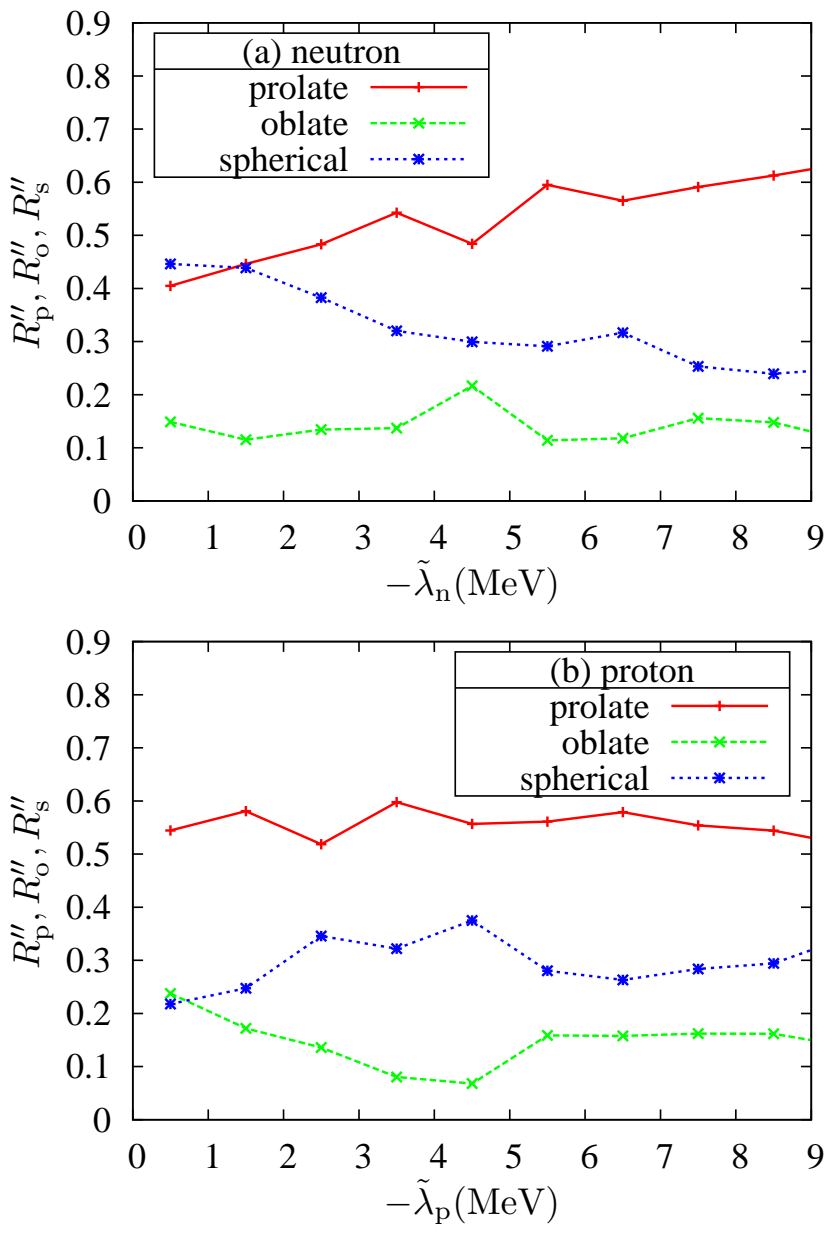

FIG. 17: Ratios of prolate, oblate, and spherical nuclei as functions of the Strutinsky smoothed Fermi energy $\tilde{\lambda}$ for the neutrons (a) or for the protons (b). The parameter set is the standard $\left(f_{l s}=f_{a}=1\right)$ universal set.

exclude it. For every one $\mathrm{MeV}$ interval of the calculated Fermi energy, we count the numbers of nuclei $N_{\mathrm{p}}, N_{\mathrm{o}}$, and $N_{\mathrm{s}}$, calculate their ratios $R_{\mathrm{p}}^{\prime \prime}, R_{\mathrm{o}}^{\prime \prime}$, and $R_{\mathrm{s}}^{\prime \prime}$, and plot the ratios with the abscissa at the center of the interval in Fig. 17] The Strutinsky smoothed Fermi energy is used as the calculated Fermi energy. There are a few other choices for the Fermi energy; for examples, half of the two nucleon separation energy, $S_{2 n} / 2\left(S_{2 p} / 2\right)$, or the chemical potential obtained by the BCS calculation. The latter is not well-defined in the case of vanishing pairing gap. The former choice has been checked that the resultant plot is very similar to Fig. 17 .

It has been sometimes discussed that in the weaklybound system the wave functions of the orbits near the Fermi surface spread out and then the shell effects play a minor role. Hence, the ratio of spherical nuclei increases in weakly-bound unstable nuclei. Such a trend is clearly seen in Fig. 17(a); the ratio of prolate nuclei decreases from 0.6 at $\tilde{\lambda}_{\mathrm{n}} \approx-8.5$ to 0.4 at $\tilde{\lambda}_{\mathrm{n}} \approx-0.5$ in balance with the increase of the spherical ratio. Even the prolate- shape dominance is inverted in the limit of weak-binding for neutrons, $-\tilde{\lambda}_{\mathrm{n}} \rightarrow 0$. As for protons, there is no such trend; the ratios are almost constants as the Fermi energy is increased and the spherical ratio of nuclei even slightly decreases. This is because the existence of the Coulomb barrier prevents the weak-binding situation even in the limit of vanishing Fermi energy.

\section{SUMMARY}

We search for the origin of the prolate-shape dominance of nuclear ground state deformation in (the combinations of) the properties of the single-particle potential for nucleons. We employ the Woods-Saxon (WS) potential and apply to it the macroscopic-microscopic theory modified for the treatment of the continuum part of the spectrum. We change the surface thickness, the strength of the spin-orbit potential, and the strength of pairing correlations to examine their influences on the ratio of prolate nuclei over more than two thousand even-even nuclei. We observe strong interference between the effects of the surface thickness and the spin-orbit potential, an especially interesting consequence of which is an oscillation versus the spin-orbit potential strength. We also find that pairing correlations enhance prolate-shape dominance.

These results are compared with the results of the Nilsson potential to elucidate some of the special features of the Nilsson potential, especially an exaggeration of the amplitude of this oscillation for the ratio of prolate nuclei.

We repeat calculations for six different parameter sets of the WS potential. We find these parameter sets can be classified according to the relation between the radii of the central and the spin-orbit potentials, which determines the effect of the surface diffuseness on spin-orbit splittings. However, as far as the behavior of the ratio of prolate nuclei concerns, different parameter sets do not change the conclusions of this paper substantially.

The difference of the definition of the ratio of prolate nuclei is also shown to be unimportant. The error in the ratio of prolate nuclei due to the truncation of the oscillator basis to express single-particle wavefunctions is shown to be sufficiently small.

Maps of ground state quadrupole deformation are shown and discussed in detail for the physics behind the ratio of prolate nuclei, which is only a single number. An example is given to illustrate the relation between the level density and the shape of the ground state in the macroscopic-microscopic theory. The ratios of prolate, oblate, and spherical nuclei are investigated also as functions of the Fermi energy. It is found that the spherical ratio increases and even exceeds the prolate ratio in the limit of weak-binding for the neutron rich unstable nuclei. 


\section{ACKNOWLEDGMENTS}

The authors would like to thank Dr. N. Onishi and Dr. K. Arita for discussions and Dr. R. Wyss for providing an unpublished parameter set "Wyss-2" for the Woods-Saxon potential. This work was supported by Grant-in-Aid for Scientific Research (C) No. 18540258 and No. 22540285 from Japan Society for the Promotion of Science. A part of the formula manipulations were carried out on the computer system at YITP in Kyoto University.
[1] A. Bohr and B.R. Mottelson, Nuclear Structure (Benjamin, New York, 1975) Vol. 2.

[2] V. M. Strutisky, Sov. J. Nul. Phys. 3, 449 (1966); Nucl. Phys. A 95, 420 (1967).

[3] H. Frisk, Nucl. Phys. A511, 309 (1990).

[4] K. Arita, arXiv:1202.5631

[5] N. Tajima and N. Suzuki, Phys. Rev. C64, 037301 (2001).

[6] S.G. Nilsson, C.F. Tsang, A. Sobiczewski, Z. Szymański, S. Wycech, C. Gustafson, I. Lamm, P. Möller and B. Nilsson, Nucl. Phys. A131, 1 (1969).

[7] S. Takahara, N. Onishi, Y. R. Shimizu, and N. Tajima, Phys. Lett. B702, 429 (2011).

[8] N. Tajima, Y. R. Shimizu, and S. Takahara, Phys. Rev. C82, 034316 (2010).

[9] A. T. Kruppa, Phys. Lett. B431, 237 (1998).

[10] T. Ono, Y.R. Shimizu, N. Tajima, S. Takahara, Phys. Rev. C82, 034310 (2010).

[11] N. Tajima, Y. R. Shimizu, and N. Suzuki, Prog. Theor. Phys. Suppl. 146, 628 (2002).

[12] R. H. Lemmer and V. F. Weisskopf, Nucl. Phys. 25, 624 (1961).

[13] K. Arita, Int. J. Mod. Phys. E, 13, 191 (2004).

[14] I. Hamamoto and B. R. Mottelson, Phys. Rev. C79, 034317 (2009).

[15] W. Zickendraht, Phys. Rev. Lett. 54, 1906 (1985).

[16] M. Brack, J. Damgård, A. S. Jensen, H. C. Pauli, V. M. Strutinsky, and C. Y. Wong, Rev. Mod. Phys. 44, 320 (1972).
[17] T. Bengtsson and I. Ragnarsson, Nucl. Phys. A436,14 (1985).

[18] R. Bengtsson, J. Dudek, W. Nazarewicz and P. Olanders, Physica Scripta 39, 196 (1989).

[19] S. Ćwiok, J. Dudek, W. Nazarewicz, J. Skalski, and T. Werner, Comput. Phys. Commun. 46, 379 (1987).

[20] J. Blomquist, S. Wahlborn, Ark, Fiz. 16, 543 (1960).

[21] E. Rost, Phys. Lett. B26, 184 (1968).

[22] V. A. Chepurnov, Yad. Fiz. 6, 955 (1967).

[23] A. Bhagwat, X. Viñas, M. Centelles, P. Schuck, and R. Wyss, Phys. Rev. C 81, 044321 (2010).

[24] R. Wyss, private communication.

[25] T. Shoji and Y. R. Shimizu, Prog. Theor. Phys. 121, 319 (2009).

[26] W. Nazarewicz, T. R. Werner, J. Dobaczewski, Phys. Rev. C 50, 2860, (1994).

[27] A. V. Afanasjev, D. B. Fossan, G. J. Lane and I. Ragnarsson, Phys. Rep. 322, 1 (1999).

[28] W. Nazarewicz, J. Dudek, R. Bengtsson, T. Bengtsson, and I. Ragnarsson, Nucl. Phys. A435, 397 (1985).

[29] N. Tajima, S. Takahara, and N. Onishi, Nucl. Phys. A603, 23 (1996).

[30] Note that the expression of the Nilsson potential in Ref. 7] has a few misprints, which are now corrected.

[31] Note that we plotted $R_{\mathrm{p}}^{\prime}$ by mistake in Fig. 1 of Ref. 7], which is consequently the same figure as Fig. 4(a), not Fig. 3(a). 